ISSN: 0514-7336

DOI: http://dx.doi.org/10.14201/zephyrus20198489113

\title{
CIRCULACIÓN Y CONSUMO DE PLOMO EN LAS ISLAS BALEARES DURANTE LA EDAD DEL HIERRO. NUEVOS DATOS ISOTÓPICOS DE GALENAS Y METALES ARQUEOLÓGICOS
}

\section{Iron Age circulation and use of lead in the Balearic Islands. New isotopic data from galena and archaeological metals}

\author{
Laura Perelló Mateo y Bartomeu Llull Estarellas \\ Dpto. de Ciencias Históricas y Teoría de las Artes. Universidad de las Islas Baleares. Ctra. de Valldemossa, km 7,5. \\ Palma (Islas Baleares). Correo-e: l.perello@uib.es; bartomeu.llull@uib.es. ID ORCID: https:/lorcid.org/0000-0002- \\ 0590-0687; https://orcid.org/0000-0002-7328-4193
}

Recepción: 5/04/2019; Revisión: 11/07/2019; Aceptación: 30/09/2019

\begin{abstract}
Resumen: En este trabajo se examinan los resultados de varias series analíticas de minerales y objetos arqueológicos procedentes de las Islas Baleares. En total, se presentan análisis de composición elemental y de isótopos de plomo de 13 muestras de galenas de depósitos de Mallorca y Menorca, así como de 18 objetos arqueológicos elaborados en plomo y en aleaciones ternarias ricas en este metal, todos procedentes de distintas necrópolis mallorquinas de cronología postalayótica (c. 550-123 a. C.). El objetivo del estudio es analizar la circulación del plomo y obtener información sobre las estrategias de gestión y consumo de este metal por parte de las comunidades locales durante la Segunda Edad del Hierro. Los datos aportados permiten realizar análisis comparativos que, junto a los ya existentes generados por otros proyectos, proporcionan una perspectiva más definida sobre la integración de las Islas Baleares en el panorama de los circuitos de intercambio mediterráneos durante estas cronologías. Los resultados también reflejan la complejidad de dichas redes, en las que el mundo fenicio-púnico tiene un papel especialmente relevante, aunque, paralelamente, se intuye el aprovechamiento de recursos locales por parte de las comunidades indígenas del archipiélago.

Palabras clave: arqueometalurgia; análisis de isótopos de plomo; postalayótico; mundo fenicio-púnico; Mediterráneo; Mallorca; minerales de plomo.

AвSTRACT: This paper examines the results of a series of analyses of minerals and archaeological objects from the Balearic Islands. In total, it presents the analytical results of the elemental and lead isotope compositions of 13 samples of galena deposits from Mallorca and Minorca, in addition to 18 archaeological objects made of lead and lead-rich ternary alloys from different Mallorcan necropolises from the Post-Talayotic period (c. 550$123 \mathrm{BC}$ ). The aim of the study is to analyse the circulation of lead and to gain an insight into strategies for the management and consumption of this metal by local communities during the Late Iron Age. With these data, comparative analyses can be made and, combined with data from other projects, a better vision can be gained on the Balearic Islands' integration in Mediterranean trade routes during this period. The results also reflect the complexity of these networks, in which the Punic-Phoenician world played a particularly important role, although, in parallel with this, the use of local resources by local native Balearic communities can be inferred.

Key words: archaeometallurgy; lead isotope analysis; Post-Talayotic; Phoenician-Punic world; Mediterranean, Mallorca; lead minerals.
\end{abstract}

(c) Universidad de Salamanca

Zephyrus, LXXXIV, julio-diciembre 2019, 89-113 


\section{Introducción ${ }^{1}$}

A partir del Bronce Final y, sobre todo, desde principios del primer milenio, se empiezan a detectar en las Islas Baleares cambios que afectan a diferentes aspectos de la materialidad cultural de las comunidades locales. Así, por ejemplo, vemos cómo cambia la concepción espacial y arquitectónica de los poblados (Calvo et al., 2009; Galmés, 2015). También se observan cambios importantes en el mundo funerario (Guerrero et al., 2007) y aparecen novedades en la tecnología metalúrgica, que debemos entender como la continuidad de las antiguas tradiciones a las que se incorporan nuevos saberes (Salvà et al., 2018).

Esto sucede en el marco del contexto general del occidente europeo, en el que se experimentan cambios a diferentes niveles y que marcarán el inicio de la Edad de Hierro. Así, en estas cronologías llegan a esta área geográfica toda una serie de técnicas metalúrgicas nuevas que acabarán siendo adoptadas por los artesanos locales. A partir de estos momentos, las aleaciones ternarias aparecerán cada vez con más frecuencia en el Mediterráneo. Se trata de nuevas

1 Los autores forman parte de Arqueouib. Grupo de investigación de cultura material y gestión del patrimonio arqueológico. La información presentada es parte de la transferencia de conocimientos de los proyectos de investigación Archipiélagos: Paisajes, comunidades prehistóricas insulares y estrategias de conectividad en el Mediterráneo Occidental. El caso de las Islas Baleares durante la Prehistoria (HAR201567211-P), financiado por el Ministerio de Economía y Competitividad, cuyo investigador principal es M. Calvo Trias (UIB). Este estudio también se ha beneficiado del programa operativo cofinanciado por el Fondo Social Europeo de ayudas para FPI concedidas por el Govern de les Illes Balears (Conselleria d'Educació, Cultura i Universitats). Asimismo, agradecemos a I. Montero Ruiz (CCHS-CSIC) su ayuda en la realización de análisis XRF, así como las valiosas aportaciones realizadas durante la codirección de la tesis Tecnología metalúrgica del cobre y del bronce durante el periodo postalayótico en Mallorca (ca. s. VI a. C.-s. I a. C.), de las que este artículo se ha visto beneficiado. También damos las gracias a J. Hernández-Gasch y a J. Merino por permitirnos analizar algunas de las piezas de Son Real y de Son Espases. Agradecemos también el apoyo técnico y humano de los Servicios Generales de Investigación (sGrker) de la UPv/EHU. recetas, consideradas de tradición atlántica, que permitirán el manejo de coladas más voluminosas y la fabricación de objetos cada vez más complejos (Montero, 2010: 175).

Durante el primer milenio, y más o menos paralelamente a la generalización de estos nuevos conocimientos, se documenta la primera siderurgia, representando la novedad tecnológica más destacada de este momento. En este caso, también nos encontramos ante una tecnología aprendida en el occidente mediterráneo, para la que, aparentemente, existirían dos vías de introducción en la Península Ibérica no excluyentes: una continental y otra, la más temprana, desde el Mediterráneo, a través de los agentes fenicios en su expansión hacia Occidente (Rovira, 2000: 215; Renzi y Rovira, 2015: 122; Guerrero et al., 2002: 228).

Los primeros objetos de hierro en el archipiélago balear para los que se tienen dataciones absolutas proceden de la necrópolis menorquina de Cova des Càrritx -Ciutadella- hacia el s. Ix a. C., si bien algunos objetos de la vecina cueva de Es Forat de Ses Aritges podrían ser un poco anteriores (Lull et al., 1999: 233). Estas cronologías estarían apuntando a una llegada de objetos de este metal procedentes de intercambios algo anteriores a la fecha histórica de la fundación de Ebussus, que correspondería a las dataciones radiocarbónicas de en torno a 800-750 a. C. (Guerrero et al., 2002: 230). También el elemento fenicio aparece vinculado a la intensificación de la explotación de minerales argentíferos en la Península Ibérica hacia el s. viII a. C. Del mismo modo, todo indica que S’Argentera -Ibiza- se empieza a minar de forma contemporánea al establecimiento de colonos en Sa Caleta -s. vII a. C.(Ramon et al., 2011; Hermanns, 2014b). En este sentido, cabe señalar que la metalurgia de la plata y del plomo deben ser consideradas conjuntamente, sobre todo a partir de estas cronologías, puesto que es en estos momentos cuando también se desarrolla una nueva y compleja técnica extractiva conocida como copelación. Con esta técnica, para la obtención de la plata a partir de minerales argentíferos con baja presencia de plomo, es necesaria la adición de este último metal. El sistema consiste en captar la 

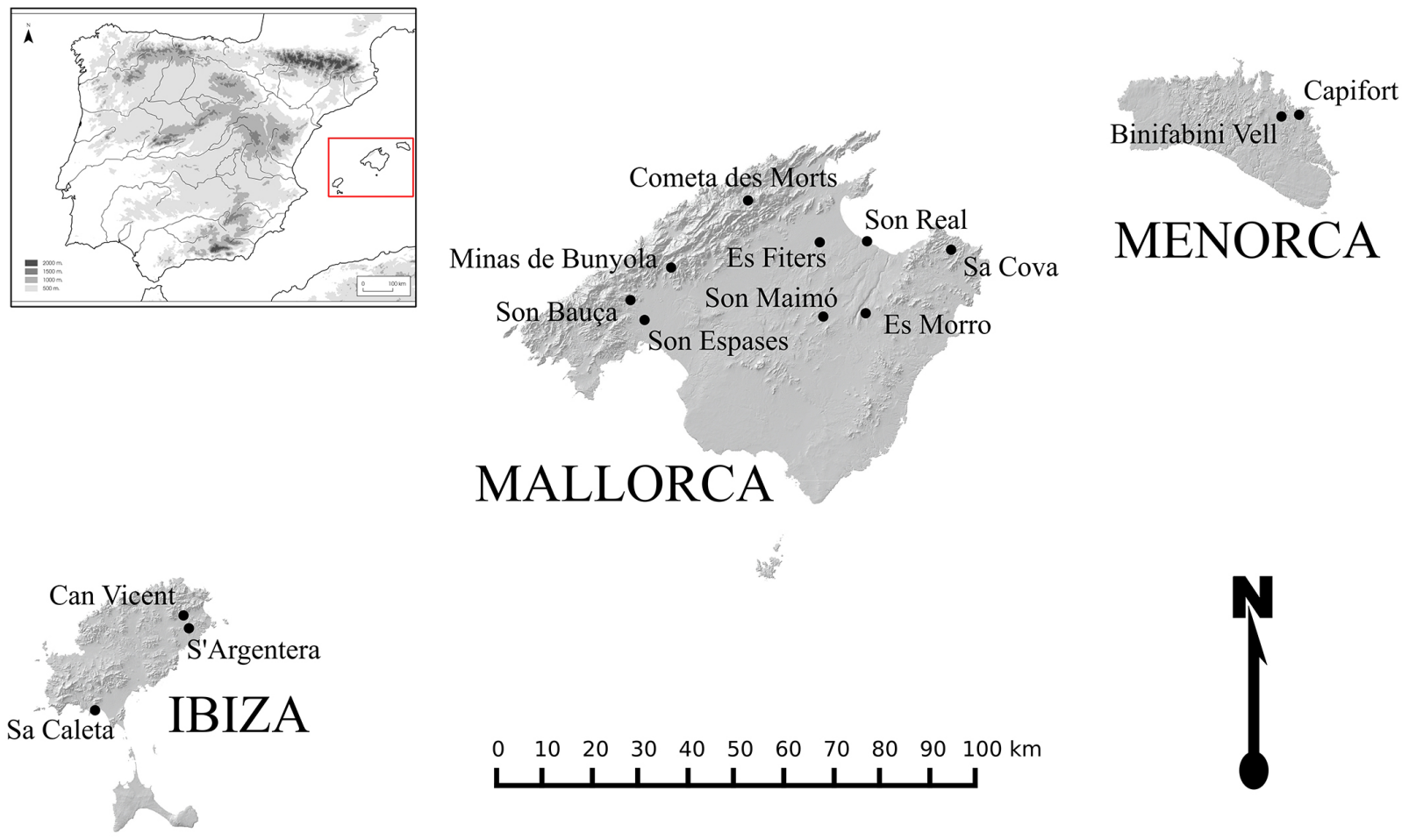

FIG. 1. Localización de los yacimientos mencionados en el texto. Mapa IDEIB, (CGovern de les Illes Balears.

plata utilizando el plomo, puesto que es más soluble en este metal que el resto de elementos. Posteriormente, para desplatar el régulo-la masa metálica de plomo y plata- se requiere un ambiente oxidante a altas temperaturas en el cual el plomo resultante del proceso es en forma de litargirio - $\mathrm{PbO}-$. En el caso de galenas argentíferas ricas en plomo no se hace necesaria la adicción de ese elemento para el proceso.

La introducción de esta técnica en la Península Ibérica está vinculada al desarrollo de la colonización fenicia. Con ello, la necesidad de plomo para llevar a cabo el proceso de copelación creó una demanda de este metal que se tradujo en un flujo importante de plomo hacia zonas donde existían recursos de plata con escaso contenido en plomo. Este es el caso del Suroeste Peninsular, donde se extrajo plata, principalmente, de jarositas argentíferas (Murillo-Barroso et al., 2016: 79). Además, este fenómeno albergó una contrapartida, ya que los subproductos generados con esta actividad extractiva pudieron acabar siendo también objeto de intercambio (Montero et al., 2008), como atestiguan las más de 400 tortas de litargirio halladas en el pecio de Mazarrón (Negueruela et al., 2000: 1677). Así es como las actividades de copelación generaron un gran tráfico de plomo, tanto procedente de las minas, como de subproductos del proceso. Seguramente, la presencia de todo este plomo puesto en circulación contribuyó de forma significativa al incremento de los bronces ternarios en toda la cuenca mediterránea (Montero, 2010: 175). En este contexto, vemos como la presencia de objetos con altos contenidos en plomo en las islas irá en aumento a medida que avanza la Edad del Hierro, y esto será notable durante el Postalayótico -c. 550123 a. C.-. Las piezas que se analizan en este trabajo se enmarcan principalmente en contextos de necrópolis de estas cronologías más recientes. El aumento de bronces ternarios ricos en plomo y la aparición de piezas fabricadas únicamente en este metal será uno de los rasgos más destacados de la metalurgia de este período. 


\section{Metodología analítica}

Para conocer la composición elemental de los metales y los minerales, se han aplicado las siguientes técnicas analíticas: Microscopio Electrónico de Barrido -SEM- equipado con una sonda basada en el efecto de Fluorescencia de Rayos x -RX-EDS-; equipo portátil de Fluorescencia de Rayos X -XRF-; y Espectrómetro de Masas por Plasma Acoplado Inductivamente -ICP-MS-. Los análisis de Isótopos de Plomo - LIA- se han utilizado para determinar posibles procedencias. La generalización de esta técnica analítica ha dado un gran impulso a los estudios de procedencias de metales no ferrosos desde principios de s. XXI, consolidándose como la principal herramienta de investigación en este campo (Montero, 2018).

\subsection{SEM $y$ RX-EDS}

Los análisis RX-EDs han sido realizados en los Servicios Científico-Técnicos de la Univ. de las Islas Baleares -UIB- bajo la supervisión del técnico F. Hierro con un Microscopio Electrónico de Barrido HITACHI S-3400N equipado con EDS BRUKER AXS GmbH y detector XFLASH 4010 con resolución de $133 \mathrm{ev}$. El equipo cuenta también con el paquete de software Quantax 400. Los análisis globales efectuados con esta técnica siempre se realizaron barriendo en ventanas relativamente grandes, generalmente de entre 100x y 200x, y al mismo tiempo barriendo zonas más pequeńas para obtener información más concreta. Se procedió a eliminar la pátina en la zona de análisis por sistema, de modo que este se efectuó sobre metal sano, salvo que se indique lo contrario en las tablas. Los resultados son cualitativos - detecta elementos desde el carbono hasta el uranio-y semicuantitativos, normalizados a 100\%. Siempre que fue posible se realizó más de un análisis por zona y objeto, debido al riesgo existente de estar analizando áreas heterogéneas de las aleaciones, en las que pudieran existir segregados o en las que pudieran aparecer sobrevalorados determinados

(C) Universidad de Salamanca elementos, por ejemplo, debido al deterioro del metal. En este sentido, poder observar con el microscopio electrónico la zona de análisis es una ventaja, ya que permite valorar si se debe discriminar alguna muestra por este motivo.

\subsection{Fluorescencia de Rayos $X$ (XRF)}

Los análisis realizados mediante esta técnica se efectuaron con el espectrómetro INNOV-X Alpha equipado con tubo de Rayos x, del Museo Arqueológico Nacional, gracias al Proyecto Arqueometalurgia de la Península Ibérica (CCHs-CsIC). Los tiempos de adquisición se fijaron en $40 \mathrm{~s}$ y los valores cuantitativos fueron calculados a partir de una calibración ajustada con patrones certificados. Los resultados van expresados como porcentaje en peso $-\mathrm{ND}=$ no detectado, $\mathrm{tr}=$ trazas - . En el caso de la plata $-\mathrm{Ag}-\mathrm{y}$ antimonio - $\mathrm{Sb}-$ el límite de detección es del 0,15\%, para el resto de elementos se sitúa en el $0,02 \%$. Los elementos ligeros - LE o ligth elements- los cuantifica cuando superan el $30 \%$ en el contenido de la muestra. Suelen aparecer si la pieza está muy corroída o mineralizada. Esta técnica permite hacer análisis elementales generales de la superficie de la muestra. Debido a la escasa capacidad de penetración, se procedió a limpiar la superficie de la muestra a fin de eliminar la pátina o contaminación superficial para analizar el metal sano siempre que fue posible, al igual que en los análisis con RX-EDS, de lo contrario se indicará en las tablas. Todos los minerales de plomo fueron analizados mediante esta técnica.

\subsection{ICP-MS}

Dos muestras de la necrópolis de Son Real, en Santa Margalida, Mallorca, han sido analizada mediante ICP-Ms en el Servicio General de Geocronología y Geoquímica Isotópica -IBERCRON- de la Univ. del País Vasco. Debido a las limitaciones de criterios de conservación, las muestras extraídas eran pequeñas, por lo que la preparación de la 
misma se realizó adecuando los protocolos habituales a las cantidades enviadas al laboratorio. Los análisis se realizaron con un espectrómetro de masas cuadrupolar con fuente de plasma acoplado inductivamente -Q-ICP-MS- marca Thermo, modelo Xseries-II, equipado con una interfase XT, antorcha apantallada y nebulizador concéntrico. La calibración del mismo se realizó a partir de soluciones multielementales de $1000 \mathrm{ppm}$ diluidas al entorno esperado para la muestra. Para el control de la deriva instrumental se utilizó como estándar interno $\mathrm{Rh}$, que es añadido online. Para el control de la calidad de los resultados se emplearon soluciones multielementales $-\mathrm{QC}-$, preparadas de manera análoga a las de la calibración.

\subsection{Isótopos de plomo}

Las muestras analizadas fueron tomadas siempre con brocas o limas nuevas, no usadas con anterioridad, debido a los altos riesgos de contaminación. Las proporciones de isótopos de plomo de los metales y minerales mallorquines y menorquines fueron medidas en el Servicio General de Geocronología y Geoquímica Isotópica -IBERCRON- de la UPV, mediante un espectrómetro de masas con fuente de plasma acoplado inductivamente de alta resolución y multicolección -MC-ICP-Ms Neptune-, para la corrección del fraccionamiento en masa se utilizó el material certificado de referencia NBS-981. Siguiendo otros trabajos (Montero, 2010: 84), se considerará que en una aleación con más del $2 \% \mathrm{~Pb}$ las analíticas están indicando con seguridad el origen del plomo y no del cobre.

\section{Distribución geográfica de recursos de mineral de plomo en las Islas Baleares y su caracterización}

Los minerales de plomo están presentes en todo el archipiélago, con la excepción de Formentera. A continuación, se describen las mineralizaciones documentadas en las Islas Baleares (Fig. 1).

(C) Universidad de Salamanca

\subsection{Mallorca}

Existe un yacimiento filoniano de cierta importancia en el término municipal de Bunyola, en una colina donde se explotó intensamente mineral de galena en época contemporánea en una mina conocida como San Mateo o mina de Son Creus. Es difícil precisar desde cuándo pudo haberse explotado este yacimiento de galena. Se tienen noticias de hallazgos casuales en algunas galerías que apuntarían a una explotación de época romana (Enseñat, 1975). De hecho, es fácil ver abundantes restos de cerámica romana de diversas cronologías concentrados en diferentes puntos de la colina, especialmente en las zonas bajas de la vertiente meridional. Así mismo, en el Museo Arqueológico de Cataluña se conserva una lucerna romana tardorrepublicana $-n .{ }^{\circ}$ de inventario 28478- Tipo g de Ricci (1973) hallada en una de sus galerías.

Por otro lado, Encinas (2014: 248) menciona el hallazgo de varios fragmentos de ánforas bajo los escombros de los rellenos de pozos internos de la mina. Por los dibujos de las piezas, podrían tratarse de ejemplares fechables hacia el s. II a. C., si bien la adscripción ebusitana que hace el autor no sería correcta, siendo una de ellas claramente grecoitálica ${ }^{2}$. En cualquier caso, la evidencia de estos materiales en el interior de las galerías podría estar indicando una explotación de la mina cronológicamente no muy lejana al momento de la conquista romana de Mallorca -123 a. C.--

En el contexto de una serie de prospecciones programadas en diversas zonas de la sierra de Tramuntana llevadas a cabo por el grupo de investigación Arqueouib, se recogieron muestras de galenas de 12 puntos del complejo minero de Bunyola, principalmente procedentes de escombreras. A nivel elemental, no se detecta $\mathrm{Ag}$ ni Sb en ninguna de las muestras analizadas (Fig. 2), por lo que de existir los niveles estarían por debajo del nivel de detección del equipo utilizado. También se detecta $\mathrm{Zn}$ de forma significativa, siempre como elemento minoritario o mayoritario. Así mismo, el $\mathrm{Cu}$ aparece en casi todos los casos como elemento minoritario. De

2 Com. pers. de C. Quintana.

Zephyrus, LXXXIV, julio-diciembre 2019, 89-113 


\begin{tabular}{|c|c|c|c|c|c|c|c|c|c|c|c|c|c|c|c|c|}
\hline & IsLA & $\begin{array}{c}\text { N. }{ }^{\circ} \text { DE } \\
\text { MUESTRA }\end{array}$ & $\begin{array}{l}\text { N. }{ }^{\circ} \text { DE } \\
\text { ANÁLISIS }\end{array}$ & Mineral & $\mathrm{Fe}$ & $\mathrm{Ni}$ & $\mathrm{Cu}$ & $\mathrm{Zn}$ & As & $\mathrm{Ag}$ & Sn & $\mathrm{Sb}$ & $\mathrm{Au}$ & $\mathrm{Pb}$ & $\mathrm{Bi}$ & $\mathrm{LE}$ \\
\hline Bunyola & Mallorca & MA11-2 & PA24394 & galena & 0,2 & ND & 0,29 & 0,61 & ND & ND & ND & ND & $\mathrm{ND}$ & 98,9 & ND & $\mathrm{ND}$ \\
\hline Bunyola & Mallorca & MA11-3 & PA24395 & galena & ND & ND & 0,32 & 0,1 & ND & ND & ND & ND & ND & 99,6 & ND & ND \\
\hline Bunyola & Mallorca & MA11-6 & PA24396 & galena & 0,71 & ND & 0,32 & 0,18 & ND & ND & ND & ND & ND & 98,8 & ND & ND \\
\hline Bunyola & Mallorca & MA11-10 & PA24397 & galena & 0,36 & $\mathrm{ND}$ & 0,21 & 0,31 & ND & ND & ND & ND & ND & 99,1 & ND & ND \\
\hline Bunyola & Mallorca & MA10-2 & PA24398 & galena & 1,54 & $\mathrm{ND}$ & 0,09 & 0,54 & ND & $\mathrm{ND}$ & ND & ND & $\mathrm{ND}$ & 20,6 & ND & 77,2 \\
\hline Bunyola & Mallorca & MA09-3 & PA24399 & galena & 9,96 & $\mathrm{ND}$ & 0,45 & 4,55 & ND & $\mathrm{ND}$ & ND & ND & ND & 85 & ND & $\mathrm{ND}$ \\
\hline Bunyola & Mallorca & MA- 8 A & PA23146 & galena & 2,6 & $\mathrm{ND}$ & 0,4 & 1,01 & $\mathrm{ND}$ & $\mathrm{ND}$ & ND & ND & $\mathrm{ND}$ & 96 & ND & $\mathrm{ND}$ \\
\hline Bunyola & Mallorca & MA-8B & PA23156 & galena & 1,7 & $\mathrm{ND}$ & ND & 1,21 & ND & ND & ND & ND & ND & 97,09 & ND & $\mathrm{ND}$ \\
\hline Bunyola & Mallorca & MA-8C & PA23155 & galena & 0,67 & ND & 0,1 & 0,42 & ND & ND & ND & ND & ND & 1,77 & ND & 96,0 \\
\hline Bunyola & Mallorca & MA-8C & PA23155b & galena & 4,12 & $\mathrm{ND}$ & ND & 1,46 & ND & $\mathrm{ND}$ & ND & ND & $\mathrm{ND}$ & 94,42 & ND & $\mathrm{ND}$ \\
\hline Capifort & Menorca & ME14-B1 & PA23159 & galena & $\mathrm{ND}$ & $\mathrm{ND}$ & ND & 20,56 & $\mathrm{ND}$ & $\mathrm{ND}$ & ND & $\mathrm{ND}$ & $\mathrm{ND}$ & 79,44 & ND & $\mathrm{ND}$ \\
\hline Capifort & Menorca & ME14-A1 & PA23160 & galena & $\mathrm{ND}$ & $\mathrm{ND}$ & ND & ND & $\mathrm{ND}$ & $\mathrm{ND}$ & ND & $\mathrm{ND}$ & $\mathrm{ND}$ & 100 & ND & $\mathrm{ND}$ \\
\hline Capifort & Menorca & ME14-A1 & PA23160 & galena & 0,55 & $\mathrm{ND}$ & $\mathrm{ND}$ & ND & ND & $\mathrm{ND}$ & ND & ND & ND & 99,45 & ND & ND \\
\hline Binifabini & Menorca & ME21 & PA23147 & galena & $\mathrm{ND}$ & $\mathrm{ND}$ & 0,2 & $\mathrm{ND}$ & $\mathrm{ND}$ & $\mathrm{ND}$ & ND & ND & $\mathrm{ND}$ & 99,8 & ND & $\mathrm{ND}$ \\
\hline Binifabini & Menorca & MC & PA21327 & galena & 1,15 & $\mathrm{ND}$ & ND & 2,64 & $\mathrm{ND}$ & $\mathrm{ND}$ & ND & $\mathrm{ND}$ & ND & 96,21 & ND & $\mathrm{ND}$ \\
\hline
\end{tabular}

FIG. 2. Análisis elemental de muestras de galena de Mallorca y Menorca que se presentan en este artículo.

todas estas muestras, se analizaron nueve mediante isótopos de $\mathrm{Pb}$ con unos resultados que nos muestran un campo isotópico bien definido, con valores muy parecidos entre ellos (Fig. 3).

Paralelamente a nuestro estudio, en una publicación se dieron a conocer otros datos isotópicos de este complejo minero (Hermanns, 2014a), presentando unos resultados completamente distintos a los obtenidos por el equipo Arqueouib. En las gráficas, se reflejan dichas diferencias mostrando para Bunyola dos grupos muy consistentes y alejados entre sí (Fig. 4). Las muestras analizadas en el trabajo de Hermanns (2014a) en las gráficas se agrupan en un espacio cercano a los valores de Túnez (Jemmali et al., 2011, 2013, 2014) (Fig. 5) y Almería (Arribas y Tosdal, 1994), aunque sin llegar a coincidir plenamente con estas regiones (Fig. 4). En cambio, las nueve muestras de galenas recogidas por el grupo Arqueouib presentan valores más altos para las ratios isotópicas ${ }^{208} \mathrm{~Pb} /{ }^{206} \mathrm{~Pb}$ y ${ }^{207} \mathrm{~Pb} /{ }^{206} \mathrm{~Pb}$ (Fig. 3) y, por lo tanto, similares a las galenas de Menorca, aunque por ahora parece que pueden

\begin{tabular}{|l|l|c|c|c|c|c|c|}
\hline \multicolumn{1}{|c|}{ ID } & MUESTRA & ${ }^{208} \mathrm{~Pb} /{ }^{206} \mathrm{~Pb}$ & ERROR $(2 \mathrm{SE})$ & ${ }^{207} \mathrm{~Pb} / 206 \mathrm{~Pb}$ & ERROR (2SE) & ${ }^{206} \mathrm{~Pb} /{ }^{204} \mathrm{~Pb}$ & ERROR (2SE) \\
\hline ME21 & Binifabini & 2,10069 & 0,00004 & 0,85489 & 0,00001 & 18,3374 & 0,0007 \\
\hline MC & Binifabini & 2,10066 & 0,00004 & 0,85530 & 0,00001 & 18,3237 & 0,0006 \\
\hline MA8A & Bunyola & 2,10301 & 0,00004 & 0,85634 & 0,00001 & 18,3107 & 0,0012 \\
\hline MA8B & Bunyola & 2,10301 & 0,00004 & 0,85635 & 0,00001 & 18,3104 & 0,0007 \\
\hline MA8C & Bunyola & 2,10284 & 0,00004 & 0,85624 & 0,00001 & 18,3122 & 0,0007 \\
\hline MA11-2 & Bunyola & 2,10291 & 0,00005 & 0,85632 & 0,00002 & 18,3092 & 0,0010 \\
\hline MA11-3 & Bunyola & 2,10281 & 0,00004 & 0,85631 & 0,00001 & 18,3092 & 0,0011 \\
\hline MA11-6 & Bunyola & 2,10287 & 0,00004 & 0,85633 & 0,00001 & 18,3086 & 0,0011 \\
\hline MA11-10 & Bunyola & 2,10278 & 0,00004 & 0,85629 & 0,00001 & 18,3090 & 0,0011 \\
\hline MA10-2 & Bunyola & 2,10186 & 0,00004 & 0,85563 & 0,00001 & 18,3243 & 0,0009 \\
\hline MA09-3 & Bunyola & 2,10289 & 0,00005 & 0,85633 & 0,00002 & 18,3079 & 0,0009 \\
\hline MEA1 & Capifort & 2,10185 & 0,00004 & 0,85478 & 0,00001 & 18,3276 & 0,0008 \\
\hline MEB1 & Capifort & 2,10231 & 0,00004 & 0,85530 & 0,00001 & 18,3128 & 0,0007 \\
\hline
\end{tabular}

Fig. 3. Valores isotópicos de las muestras de galena de Mallorca y Menorca que se presentan en este artículo. 


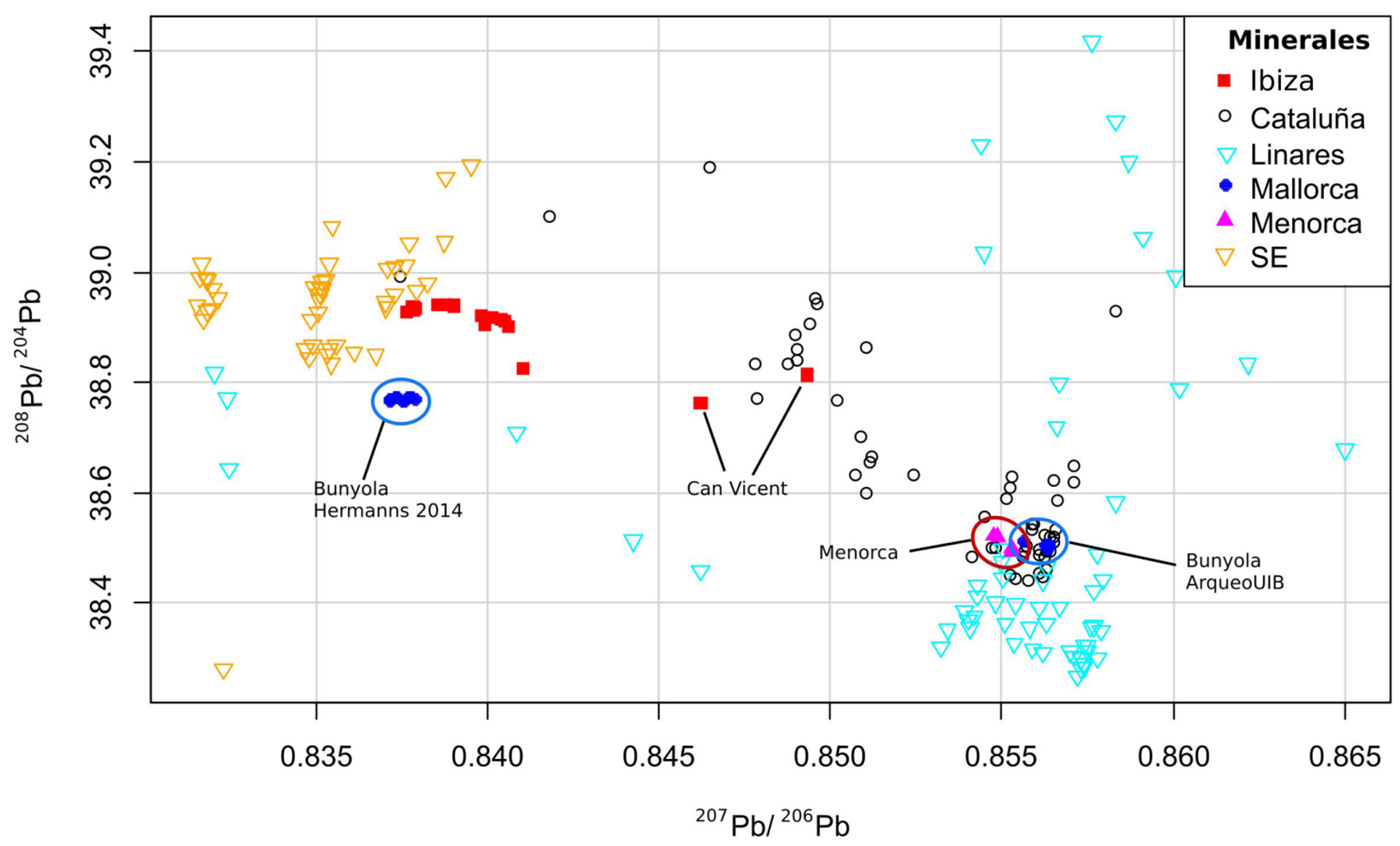

FIG. 4. Minerales de plomo de las Islas Baleares en contraste con minerales de otras regiones geográficamente cercanas. Pueden observarse las diferencias entre los dos conjuntos de muestras procedentes de Bunyola.

diferenciarse bien ambos campos isotópicos (Fig. 4). En este sentido, hay que decir que, cuando contrastamos nuestros análisis con los de otras mineralizaciones de la Península Ibérica, podemos comprobar que presentan un solapamiento importante con muestras procedentes de Cataluña (Fig. 4), y concretamente de Tarragona (Canals y Cardellach, 1997; Montero et al., 2009; Montero, 2017). Esto supondrá una dificultad añadida cuando se trate de discriminar la procedencia de ciertos metales con valores isotópicos parecidos.

Las diferencias planteadas entre las dos series de analíticas de Bunyola podrían tener varias explicaciones. En primer lugar, podríamos deducir que nos encontramos ante dos mineralizaciones muy bien definidas, con lo cual no se trataría de un depósito anómalo con una huella isotópica amplia. Más bien estaríamos ante dos filones de distinta edad geológica, coincidentes geográficamente. Sin embargo, por las descripciones del trabajo de Hermanns (2014a: 191), parece deducirse que las muestras recogidas son coincidentes con el mismo punto de recogida de muestras que nosotros hemos denominado serie MA09 (Fig. 3). En segundo lugar, al haberse realizado las dos baterías de analíticas en laboratorios diferentes, existe la posibilidad de deberse a un problema generado durante la realización de los análisis o bien por contaminación de las muestras. Siendo los resultados de nuestras muestras más coherentes con valores de las Baleares que aquí estudiamos, y a la espera de aclarar el porqué de estas diferencias isotópicas entre ambos conjuntos, en este trabajo obviaremos por el momento los datos de Hermanns (2014a) en las siguientes gráficas. En cualquier caso, podemos adelantar que por ahora no se ha observado ninguna coincidencia entre estos y los valores de mineralizaciones y metales arqueológicos de las Baleares.

\subsection{Menorca}

Para Menorca, contamos con análisis de isótopos de plomo de dos yacimientos polimetálicos: Binifabini Vell (Mercadal) y Capifort (Maó). La zona de 


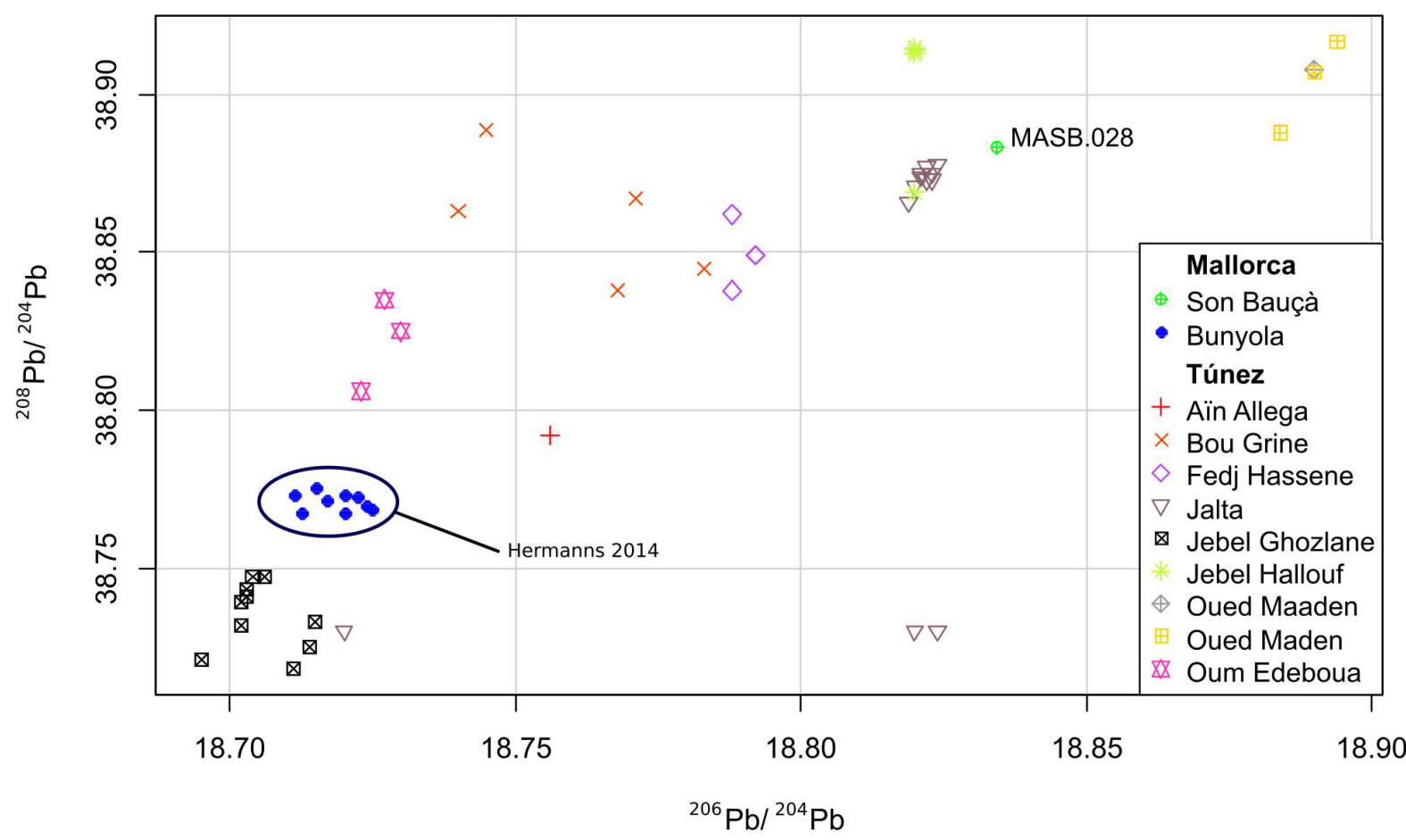

FIG. 5. Conjunto de muestras de Bunyola analizadas por Hermanns, contrastadas con diferentes mineralizaciones de Túnez; aunque estos análisis de Bunyola pueden mostrar cierto parecido con las muestras del $N$ de África, el conjunto aparece bien definido y no existe solapamiento entre minerales de una región y otra.

Binifabini Vell también fue prospectada por parte de nuestro equipo (Salvà et al., 2010; Llull y Perelló, 2013), constatando la existencia de un complejo minero de cierta entidad con labores de momentos diferenciados, aunque la adscripción cronológica de los mismos es muy compleja. Tal y como sucede en las minas de Son Creus, en Bunyola, Mallorca, sabemos por la documentación histórica que en esta zona se benefició mineral de plomo en época contemporánea (Llull y Perelló, 2013). Por otra parte, a nivel arqueológico, un simple análisis superficial permite revelar la presencia de trabajos planificados superpuestos a labores anteriores de desarrollo orgánico. Así, en este complejo minero, se documentan socavones de exploración con huellas de barrenos y de piquetas de hierro, pero también, a veces compartiendo el mismo espacio, existen labores a cielo abierto y galerías muy irregulares de cronología incierta. Destaca una de las galerías de época contemporánea que atravesó un corredor más antiguo, en el que se pueden apreciar trabajos a diferentes alturas que presentan un desarrollo orgánico, siguiendo claramente la veta de cobre. En esta zona de la mina, también es posible apreciar cómo las labores más modernas ensancharon una galería más estrecha que desemboca en una pequeña sala de planta irregular. Desafortunadamente, no se han hallado evidencias arqueológicas que permitan datar estos trabajos más antiguos. La mineralización se encuentra dentro de materiales del Triásico, el depósito es de tipo alpino con una paragénesis $\mathrm{Cu}-\mathrm{Pb}-\mathrm{Zn}$ con contenidos en As y $\mathrm{Ba}$ en algunas muestras en forma de elemento minoritario e incluso mayoritario.

La otra zona de muestreo, la de Capifort en Maó, es una explotación de cronología contemporánea que según la información encontrada en los registros del Distrito de Minas de las Baleares -Arxiu del Regne de Mallorca- se benefició con distintos nombres: Catalina (1861), Diana (1875), Luz (1883) y Enriqueta (1899), siendo la última fecha de renuncia la del año 1905 realizada por la Sociedad Cobres de Menorca $-B O E$ n. ${ }^{\circ}$ 5963, de 


\begin{tabular}{|c|c|c|c|c|c|c|c|c|c|c|c|c|c|c|c|}
\hline YaCimiEnto & ID PIEZA & ID ANÁLISIS & TIPO DE PIEZA & $\begin{array}{c}\text { Tipo DE } \\
\text { ANALíticA } \\
\end{array}$ & $\mathrm{Fe}$ & $\mathrm{NI}$ & $\mathrm{Cu}$ & $\mathrm{ZN}_{\mathrm{N}}$ & As & AG & $\mathrm{SN}_{\mathrm{N}}$ & $\mathrm{SB}_{\mathrm{B}}$ & $\mathrm{Au}$ & Рв & OBSERVACIONES \\
\hline Cometa des Morts & MACM1.051a & МАСм1.051a/01 & disco de tintinnabula & RX-EDS & ND & ND & 84,98 & $\mathrm{ND}$ & ND & ND & 9,95 & $\mathrm{ND}$ & $\mathrm{ND}$ & 5,08 & \\
\hline Cometa des Morts & MACM $1.051 \mathrm{a}$ & МАСм $1.051 \mathrm{a} / 02$ & disco de tintinnabula & RX-EDS & ND & $\mathrm{ND}$ & 81,43 & $\mathrm{ND}$ & ND & ND & 14,27 & ND & $\mathrm{ND}$ & 4,31 & \\
\hline Cometa des Morts & MACM1.051a & PA0746A & disco de tintinnabula & XRF & 0,37 & 0,13 & 81,59 & ND & ND & 0,01 & 11,53 & 0,06 & $\mathrm{ND}$ & 5,40 & \\
\hline Cometa des Morts & MACM1.083 & PA23125 & disco de tintinnabula & XRF & ND & ND & 65,80 & ND & ND & 0,23 & 4,68 & 0,24 & ND & 29,05 & mineralizado \\
\hline Es Morro & MAEM.009 & MAEM.009/01 & disco de tintinnabula & RX-EDS & ND & ND & 92,01 & ND & ND & ND & 4,67 & ND & ND & 3,32 & \\
\hline Es Morro & MAEM.009 & MAEM.009/02 & disco de tintinnabula & RX-EDS & ND & ND & 96,55 & ND & ND & ND & 2,18 & ND & ND & 1,27 & \\
\hline Es Morro & MAEM.009 & PA21593B & disco de tintinnabula & XRF & $\mathrm{ND}$ & ND & 93,00 & $\mathrm{ND}$ & $\mathrm{ND}$ & $\mathrm{ND}$ & 2,40 & $\mathrm{ND}$ & $\mathrm{ND}$ & 4,59 & \\
\hline Sa Cova & MASCO.018 & MASC0.018/01 & disco de tintinnabula & RX-EDS & ND & ND & 74,15 & ND & ND & ND & 20,55 & $\mathrm{ND}$ & $\mathrm{ND}$ & 5,3 & no alma \\
\hline Sa Cova & MASCO.018 & MASCO.018/02 & disco de tintinnabula & RX-EDS & ND & ND & 80,5 & ND & $\mathrm{ND}$ & ND & 16,09 & ND & ND & 3,41 & no alma \\
\hline Es Fiters & MAEF.014a & MAEF.014a/02 & disco de tintinnabula & RX-EDS & ND & \begin{tabular}{|l|}
$\mathrm{ND}$ \\
\end{tabular} & 97,54 & ND & $\mathrm{ND}$ & $\mathrm{ND}$ & 2,78 & $\mathrm{ND}$ & ND & 2,90 & \\
\hline Es Fiters & MAEF.014a & MAEF.014a/03 & disco de tintinnabula & RX-EDS & ND & ND & 98,54 & ND & ND & ND & 2,51 & $\mathrm{ND}$ & $\mathrm{ND}$ & 1,42 & \\
\hline Es Fiters & MAEF.014a & PA23077 & disco de tintinnabula & XRF & 0,24 & $\mathrm{ND}$ & $\mathrm{ND}$ & ND & $\mathrm{ND}$ & ND & 0,13 & $\mathrm{ND}$ & ND & 1,21 & mineralizado \\
\hline Es Fiters & MAEF.014a & PA23077P & disco de tintinnabula & XRF & 0,30 & 0,06 & 93,30 & ND & $\mathrm{ND}$ & ND & 2,92 & ND & $\mathrm{ND}$ & 3,43 & pátina \\
\hline Es Fiters & MAEF. $015 \mathrm{a}$ & MAEF.015a/01 & disco de tintinnabula & RX-EDS & $\mathrm{ND}$ & ND & 61,53 & ND & $\mathrm{ND}$ & ND & 1,55 & ND & ND & 36,92 & \\
\hline Es Fiters & MAEF.015a & MAEF.015a/02 & disco de tintinnabula & RX-EDS & ND & ND & 64,71 & ND & $\mathrm{ND}$ & ND & 4,36 & ND & ND & 30,94 & \\
\hline Es Fiters & MAEF.015 & MAEF. $015 \mathrm{a} / 03$ & disco de tintinnabula & RX-EDS & ND & $\mathrm{ND}$ & 59,21 & ND & $\mathrm{ND}$ & ND & 1,79 & $\mathrm{ND}$ & $\mathrm{ND}$ & 39,00 & \\
\hline Es Fiters & MAEF.015a & PA23097 & disco de tintinnabula & XRF & ND & ND & 76,60 & ND & $\mathrm{ND}$ & ND & 0,97 & $\mathrm{ND}$ & $\mathrm{ND}$ & 22,44 & \\
\hline Es Fiters & MAEF.016a & MAEF016a/01 & disco de tintinnabula & RX-EDS & ND & $\mathrm{ND}$ & 83,28 & ND & $\mathrm{ND}$ & ND & 14,41 & $\mathrm{ND}$ & $\mathrm{ND}$ & 2,31 & \\
\hline Es Fiters & MAEF.016a & MAEF.016a/02 & disco de tintinnabula & RX-EDS & ND & $\mathrm{ND}$ & 84,11 & ND & $\mathrm{ND}$ & ND & 13,86 & $\mathrm{ND}$ & $\mathrm{ND}$ & 2,03 & \\
\hline Es Fiters & MAEF.016a & MAEF.016a/03 & disco de tintinnabula & RX-EDS & ND & $\mathrm{ND}$ & 88,52 & ND & $\mathrm{ND}$ & $\mathrm{ND}$ & 9,28 & $\mathrm{ND}$ & $\mathrm{ND}$ & 2,20 & \\
\hline Es Fiters & MAEF.016a & PA23078 & disco de tintinnabula & XRF & ND & ND & 90,50 & ND & ND & ND & 8,28 & ND & ND & 1,20 & \\
\hline Es Fiters & MAEF.016a & PA23078P & disco de tintinnabula & XRF & 0,90 & ND & 90,60 & ND & ND & ND & 5,69 & ND & ND & 3,65 & pátina \\
\hline Es Fiters & MAEF.017a & PA23142A & disco de tintinnabula & $\mathrm{XRF}$ & 0,16 & ND & 76,00 & ND & 1,02 & ND & 0,63 & $\mathrm{ND}$ & $\mathrm{ND}$ & 22,23 & \\
\hline Es Fiters & MAEF.018 & PA23145 & varilla & XRF & 0,45 & ND & $\mathrm{ND}$ & ND & ND & ND & 2,56 & ND & ND & 3,42 & \\
\hline Son Bauçà & MASB.026a & MASB.026a/01 & campanita & RX-EDS & ND & ND & 78,48 & ND & $\mathrm{ND}$ & ND & 6,01 & ND & ND & 15,51 & \\
\hline Son Bauçà & MASB.026a & MASB.026a/02 & campanita & RX-EDS & ND & ND & 86,49 & ND & $\mathrm{ND}$ & ND & 5,34 & $\mathrm{ND}$ & $\mathrm{ND}$ & 8,17 & \\
\hline Son Bauçà & MASB.026a & MASB. $026 \mathrm{a} / 03$ & campanita & RX-EDS & ND & $\mathrm{ND}$ & 41,79 & ND & $\mathrm{ND}$ & ND & 0,00 & $\mathrm{ND}$ & $\mathrm{ND}$ & 29,79 & pátina \\
\hline Son Bauçà & MASB. $027 \mathrm{a}$ & MASB. $027 \mathrm{a} / 01$ & campanita & RX-EDS & ND & ND & 84,62 & ND & ND & ND & 12,88 & ND & ND & 2,50 & \\
\hline Son Bauçà & MASB. $027 \mathrm{a}$ & MASB. $027 \mathrm{a} / 02$ & campanita & RX-EDS & ND & ND & 82,65 & $\mathrm{ND}$ & ND & ND & 15,16 & ND & $\mathrm{ND}$ & 2,19 & \\
\hline Son Bauçà & MASB. $027 \mathrm{a}$ & MASB. $027 \mathrm{a} / 03$ & campanita & RX-EDS & ND & $\mathrm{ND}$ & 77,71 & ND & $\mathrm{ND}$ & ND & 4,11 & ND & ND & 18,18 & pátina \\
\hline Son Bauçà & MASB.028a & MASB.028a/01 & campanita & RX-EDS & ND & $\mathrm{ND}$ & 91,33 & ND & $\mathrm{ND}$ & ND & 4,70 & $\mathrm{ND}$ & $\mathrm{ND}$ & 3,97 & \\
\hline Son Bauçà & MASB.028a & MASB.028a/02 & campanita & RX-EDS & ND & ND & 94,83 & ND & $\mathrm{ND}$ & ND & 1,44 & $\mathrm{ND}$ & $\mathrm{ND}$ & 3,72 & \\
\hline Son Bauçà & MASB.028a & MASB.028a/03 & campanita & RX-EDS & ND & ND & 86,89 & ND & ND & ND & 5,85 & $\mathrm{ND}$ & $\mathrm{ND}$ & 7,26 & pátina \\
\hline Son Bauçà & MASB.029a & MASB.029a/01 & campanita & RX-EDS & ND & $\mathrm{ND}$ & 73,64 & ND & $\mathrm{ND}$ & ND & 20,27 & $\mathrm{ND}$ & $\mathrm{ND}$ & 6,10 & mineralizado \\
\hline Son Bauçà & MASB.029a & MASB.029a/02 & campanita & RX-EDS & ND & $\mathrm{ND}$ & 56,93 & ND & $\mathrm{ND}$ & $\mathrm{ND}$ & 35,37 & $\mathrm{ND}$ & $\mathrm{ND}$ & 7,69 & mineralizado \\
\hline Son Bauçà & MASB.029a & MASB. $029 \mathrm{a} / 03$ & campanita & RX-EDS & ND & ND & 63,06 & ND & ND & ND & 34,02 & ND & ND & 2,92 & pátina \\
\hline Cova de Son Maimó & MACSM.001 & PA23725 & doble hacha & XRF & ND & ND & 65,90 & ND & ND & ND & 5,27 & ND & ND & 28,80 & \\
\hline Cova de Son Maimó & MACSM.001 & PA23725P & doble hacha & XRF & 0,49 & ND & 72,90 & ND & $\mathrm{ND}$ & ND & 9,15 & $\mathrm{ND}$ & $\mathrm{ND}$ & 17,40 & \\
\hline Son Espases & MASE.001 & PA23128 & plaquita de $\mathrm{Pb}$ & XRF & 0,18 & ND & $\mathrm{ND}$ & ND & $\mathrm{ND}$ & ND & $\mathrm{ND}$ & $\mathrm{ND}$ & $\mathrm{ND}$ & 99,82 & \\
\hline Son Espases & MASE.002 & PA23126 & plaquita de $\mathrm{Pb}$ & XRF & 0,48 & ND & 0,1 & ND & ND & ND & 1,25 & ND & ND & 98,17 & \\
\hline Son Espases & MASE.002 & PA23126B & plaquita de $\mathrm{Pb}$ & XRF & 0,54 & ND & ND & ND & $\mathrm{ND}$ & ND & 1,26 & $\mathrm{ND}$ & $\mathrm{ND}$ & 98,21 & \\
\hline Son Espases & \begin{tabular}{|l|} 
MASE. 003 \\
\end{tabular} & PA23127 & plaquita de $\mathrm{Pb}$ & XRF & 1,12 & ND & 0,1 & $\mathrm{ND}$ & $\mathrm{ND}$ & ND & $\mathrm{ND}$ & $\mathrm{ND}$ & $\mathrm{ND}$ & 98,77 & \\
\hline Son Espases & MASE.004 & PA23129 & plaquita de $\mathrm{Pb}$ & XRF & 0,29 & ND & $\mathrm{ND}$ & ND & ND & ND & $\mathrm{ND}$ & ND & $\mathrm{ND}$ & 99,71 & \\
\hline
\end{tabular}

FIG. 6. Análisis elemental mediante XRF y XR-EDS de los metales estudiados en este trabajo. Algunos de los que aparecen en esta tabla no han sido analizados mediante isótopos de plomo. 
30/03/1905 art. 737-. La bocamina es inaccesible a causa de los derrumbes y, por tanto, no podemos saber de la existencia de trabajos anteriores, aunque, por la toponimia del lugar que aparece en las primeras solicitudes - Tanca del Vernís, que podríamos traducir al castellano como 'cercado del barniz'-, se deduce una utilización anterior del plomo del lugar como esmalte cerámico. Esta mineralización se encuentra entre materiales pertenecientes al Carbonífero, con galenas de paragénesis $\mathrm{Pb}-\mathrm{Zn}$ en las que no se detecta Ag ni Sb (Fig. 2).

Los valores isotópicos de ambos depósitos menorquines y los de Mallorca -Bunyola, ArqueouiB-son próximos entre sí, aunque la escasez de muestras de galena analizadas procedentes de Menorca nos obliga a ser prudentes. Sin embargo, con los datos actuales, podemos decir que, al igual que ocurre con el grupo de muestras de Bunyola-ArqueouiB-, cuando contrastamos estos análisis con los de otras mineralizaciones de la Península Ibérica, observamos solapamiento con muestras procedentes de Cataluña, especialmente de Tarragona (Canals y Cardellach 1997; Montero et al., 2009; Montero, 2017).

\subsection{Ibiza}

En cuanto a las Pitiusas, existen análisis de plomo publicados procedentes de dos afloramientos distintos en Ibiza, aunque próximos entre sí: S’Argentera y Can Vicent (Ramon et al., 2011; Hermanns, 2015) $)^{3}$. El afloramiento del Puig de S'Argentera, en el no de Ibiza a unos $5 \mathrm{~km}$ de Santa Eulàlia des Riu, es el que más nos interesa a nivel arqueológico. En este lugar se localiza un complejo minero investigado desde 2010 por un equipo liderado por el Dr. Hermanns. Si bien resulta complicado confirmar una explotación de época antigua desde el estudio directo del complejo minero, las intervenciones arqueológicas han permitido detectar la aplicación de fire-setting y la utilización de herramientas romas (Hermanns, 2014b: 270), lo que permitiría intuir, con mucha prudencia, trabajos al menos prerromanos en algunas galerías. Por otra parte, los resultados de análisis de isótopos de plomo que permitieron relacionar los subproductos metalúrgicos del yacimiento de Sa Caleta -ss. VIIvi a. C.- con la galena de S'Argentera confirmaron indirectamente una datación prerromana de las labores antiguas de estas minas (Ramon et al., 2011; Hermanns, 2014b: 309; Hermanns, 2015: 276).

Los análisis XRF publicados por Ramon et al. (2011) muestran una galena argentífera con valores altos de $\mathrm{Ag}$ y $\mathrm{Sb}$, rasgo que la diferencia del plomo de Mallorca y Menorca. Se trata de galenas muy probablemente beneficiadas para obtener plata en la antigüedad. Los mismos autores publicaron cuatro análisis isotópicos de muestras de galena argentífera procedentes de esta mina, señalando que existía un solapamiento parcial entre los campos isotópicos de S’Argentera y las minas de la Sierra de Cartagena. La publicación de Hermanns (2014b) de catorce nuevos análisis isotópicos de S’Argentera definió con más precisión el campo isotópico de Ibiza, haciendo posible una mejor discriminación del de Cartagena. En el mismo trabajo se publicaron también dos muestras de Can Vicent, un yacimiento minero que se encuentra a unos $7 \mathrm{~km}$ de S'Argentera. El investigador señala que, a pesar de la cercanía, ambos yacimientos presentan ratios isotópicas diferenciadas (Fig. 4),

\begin{tabular}{|c|c|c|c|c|c|c|c|c|c|c|c|c|c|c|c|c|c|c|c|c|c|c|c|c|}
\hline ID & $\mathrm{Na}$ & $\mathrm{Mg}$ & $\mathrm{Al}$ & $\mathrm{P}$ & $\mathrm{K}$ & $\mathrm{Ca}$ & $\mathrm{Sc}$ & $\mathrm{Ti}$ & $\mathrm{V}$ & $\mathrm{Cr}$ & $\mathrm{Fe}$ & $\mathrm{Mn}$ & $\mathrm{Co}$ & $\mathrm{Ni}$ & $\mathrm{Cu}$ & $\mathrm{Zn}$ & $\mathrm{As}$ & $\mathrm{Se}$ & $\mathrm{Rb}$ & $\mathrm{Sr}$ & $\mathrm{Y}$ & $\mathrm{Ag}$ & $\mathrm{Cd}$ & $\mathrm{In}$ \\
\hline SR14 & 7,9 & 13,9 & 10,4 & 8,8 & 753,3 & nd & nd & 1,16 & nd & nd & 10 & nd & nd & 46,21 & 54580 & 21,50 & 34,35 & nd & nd & nd & nd & nd & nd & nd \\
\hline SR15 & nd & 17,7 & nd & nd & nd & 36,8 & nd & 6,90 & 0,48 & 0,48 & nd & 0,08 & 0,11 & 0,22 & 531 & 9,22 & 0,98 & 2,27 & 0,00 & 0,30 & 0,05 & 273 & 0,05 & 0,35 \\
\hline
\end{tabular}

FIG. 7. Análisis elemental mediante ICP-MS de las dos piezas de plomo del yacimiento de Son Real. Valores expresados en ppm ( $n d=$ no detectado).

3 Ruiz de Smedt, M. C.: Metalurgia de época fenicia en Ibiza, el yacimiento de Sa Caleta. Tesis de licenciatura inédita presentada en 1992 en la Univ. de Barcelona. aunque hay que ser prudentes y esperar a contar con más análisis para tener las mineralizaciones ibicencas mejor definidas (Hermanns, 2015: 276). 


\section{Análisis de los datos elementales y de isótopos de plomo de metales y minerales}

\subsection{Metales estudiados}

Los materiales que presentamos en este trabajo se analizaron en el contexto de un proyecto de tesis ${ }^{4}$, para el cual se estudiaron objetos arqueológicos de base cobre procedentes de varias necrópolis postalayóticas $-c$. 550 a. C.-123 a. C.- de la isla de Mallorca (Fig. 1). Debido a que el objetivo del presente estudio es analizar la circulación y obtener datos sobre el consumo de plomo de las comunidades locales, hemos seleccionado varios bronces ternarios ricos en plomo, entre los que se encuentran siete discos de tintinnabula, una varilla de tintinnabula, una doble hacha votiva, un cuerno y cuatro campanillas, todos ellos recuperados en excavaciones antiguas y, por ello, sin un contexto claro. Para completar el estudio, se han ańadido análisis de seis objetos manufacturados en plomo (Figs.
6-8), todos procedentes de Mallorca y de excavaciones recientes -Son Real y Son Espases-. Salvo en el caso de las piezas de Son Espases, en Palma, el resto de piezas proceden de necrópolis indígenas.

Los únicos ejemplares que no representan tipologías exclusivamente locales son las campanitas de Son Bauçà, en Palma, puesto que son objetos muy extendidos en el mundo mediterráneo y, de hecho, son abundantes en las necrópolis fenicias (Fig. 9). En el caso de los discos tintinnabula y la varilla, se trata de objetos que solo existen en Mallorca. Son discos de metal, normalmente de bronce y generalmente asociados a percutores y varillas, por lo que se han vinculado a la producción de sonidos o de música en contextos funerarios (Perelló y Llull, 2019). La cronología para estos objetos estaría entre los ss. vi y I a. C. aproximadamente y, en el presente estudio, se analizan piezas procedentes de Cometa des Morts I (Escorca), Sa Cova (Artà), Es Morro (Manacor) y Es Fiters (Muro) (Figs. 9 y 10).

\begin{tabular}{|l|l|c|c|c|c|c|c|}
\hline \multicolumn{1}{|c|}{ Id } & \multicolumn{1}{|c|}{ MUESTRA } & ${ }^{208} \mathrm{~Pb} /{ }^{206} \mathrm{~Pb}$ & ERROR (2SE) & ${ }^{207} \mathrm{~Pb} /{ }^{206} \mathrm{~Pb}$ & ERROR (2SE) & ${ }^{206} \mathrm{~Pb} / 204 \mathrm{~Pb}$ & ERROR (2SE) \\
\hline MACMI.083 & Cometa des Morts & 2,08944 & 0,00003 & 0,84618 & 0,00001 & 18,54174 & 0,00079 \\
\hline MACMI.051 & Cometa des Morts & 2,08770 & 0,00004 & 0,84640 & 0,00001 & 18,53103 & 0,00085 \\
\hline MAEM.009 & Es Morro & 2,08273 & 0,00003 & 0,84090 & 0,00001 & 18,66805 & 0,00068 \\
\hline MAEF.014 & Es Fiters & 2,08307 & 0,00004 & 0,84199 & 0,00001 & 18,62955 & 0,00090 \\
\hline MAEF.015 & Es Fiters & 2,09600 & 0,00004 & 0,84986 & 0,00001 & 18,46131 & 0,00089 \\
\hline MAEF.016 & Es Fiters & 2,09241 & 0,00004 & 0,84858 & 0,00001 & 18,47015 & 0,00074 \\
\hline MAEF.017A & Es Fiters & 2,10202 & 0,00004 & 0,85601 & 0,00001 & 18,32711 & 0,00071 \\
\hline MAEF.018 & Es Fiters & 2,06779 & 0,00004 & 0,83477 & 0,00001 & 18,80027 & 0,00084 \\
\hline MACSM.001 & Son Maimó & 2,07622 & 0,00004 & 0,83623 & 0,00001 & 18,76862 & 0,00080 \\
\hline MASE.001 & Son Espases & 2,08424 & 0,00004 & 0,83785 & 0,00001 & 18,72991 & 0,00072 \\
\hline MASE.004 & Son Espases & 2,08660 & 0,00004 & 0,84051 & 0,00001 & 18,66787 & 0,00068 \\
\hline MASB.026 & Son Bauçà & 2,09491 & 0,00005 & 0,85170 & 0,00001 & 18,37141 & 0,00096 \\
\hline MASB.027 & Son Bauçà & 2,10087 & 0,00004 & 0,85533 & 0,00001 & 18,32791 & 0,00093 \\
\hline MASB.028 & Son Baçà̀ & 2,06447 & 0,00004 & 0,83306 & 0,00001 & 18,83444 & 0,00098 \\
\hline MASB.029 & Son Bauçà & 2,10487 & 0,00005 & 0,85667 & 0,00002 & 18,25164 & 0,00090 \\
\hline MASCO.018 & Sa Cova & 2,10269 & 0,00004 & 0,85504 & 0,00001 & 18,31253 & 0,00081 \\
\hline SR14 & Son Real & 2,08418 & 0,00004 & 0,83794 & 0,00001 & 18,72701 & 0,00081 \\
\hline SR15 & Son Real & 2,08389 & 0,00004 & 0,83942 & 0,00001 & 18,69933 & 0,00070 \\
\hline
\end{tabular}

Fig. 8. Valores isotópicos de los metales estudiados en este trabajo procedentes de: Cometa des Morts I, Es Morro, Es Fiters, Cova de Son Maimó, Son Espases, Son Bauçà, Sa Cova y Son Real.

4 Perelló, L.: Tecnología metalúrgica del cobre y del bronce durante el periodo Postalayótico en Mallorca (ca. ss. VI-I a. C.). Tesis doctoral inédita presentada en 2017 en la Univ. de les Illes Balears.
En cuanto a las plaquitas de plomo, también pueden encontrarse en Menorca. Son unos de los objetos más característicos y abundantes de las necrópolis postalayóticas (Enseñat, 1975). Se trata 


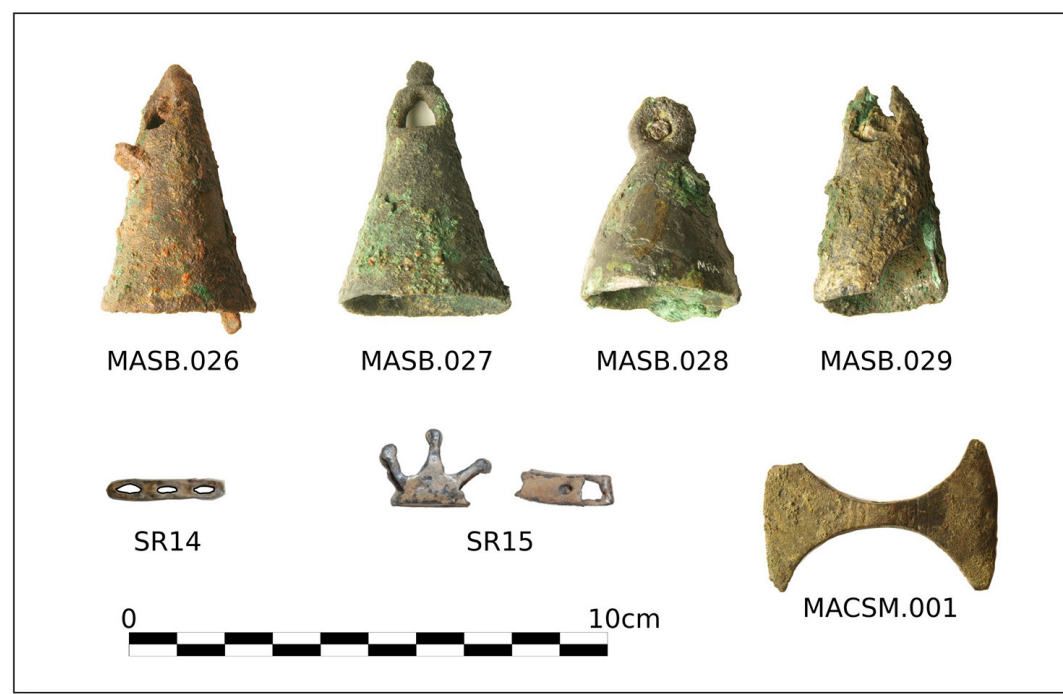

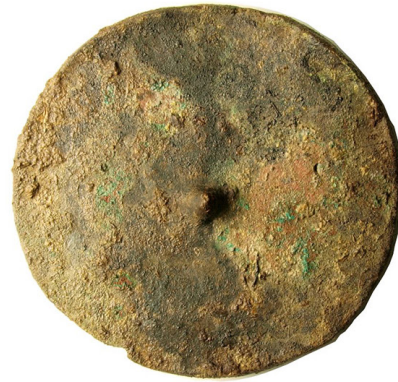

MASCO.018

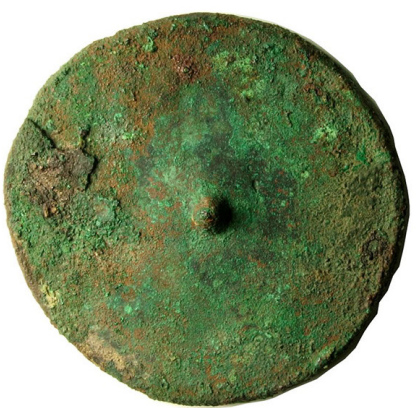

MAEF.014

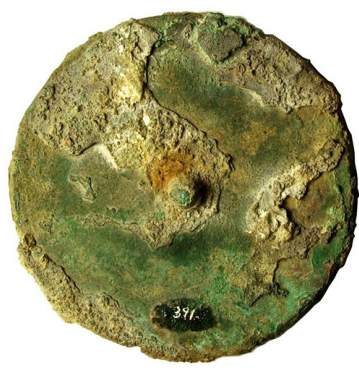

MAEM.009

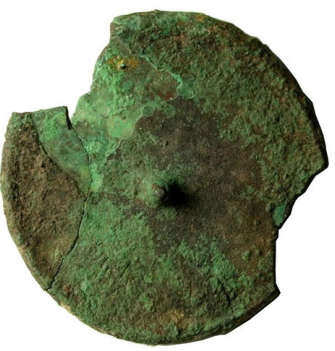

MAEF.015

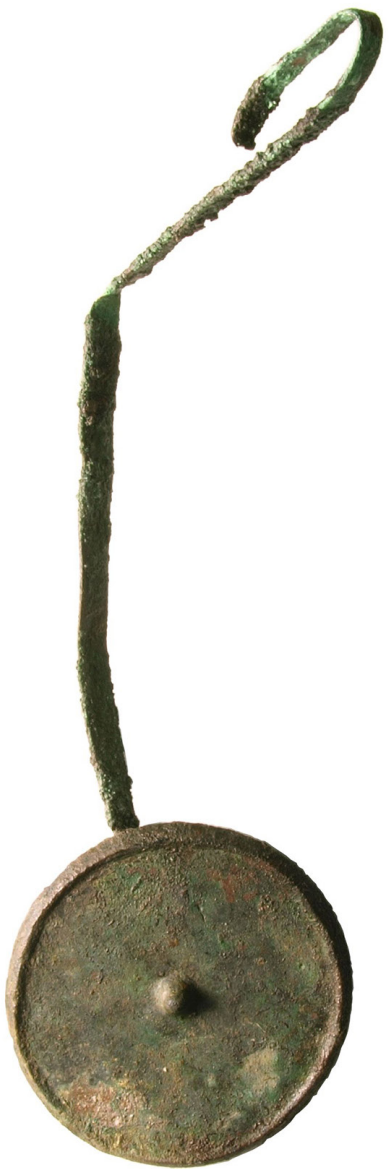

MAEF.017

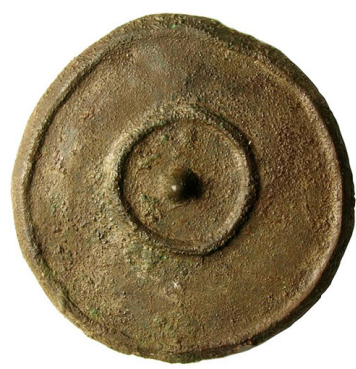

MAEF.016
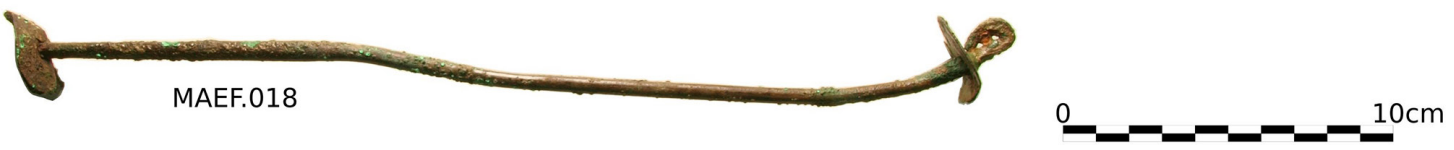

FIG. 9. Imagen de algunos de los metales analizados en este trabajo; arriba a la izquierda: campanitas, hacha bipenne, placa troquelada y objeto indefinido de plomo; el resto son discos y varillas de tintinnabula (imágenes SR 14 y SR15 tomadas de Hernández-Gasch et al., op. cit. n. 7). 

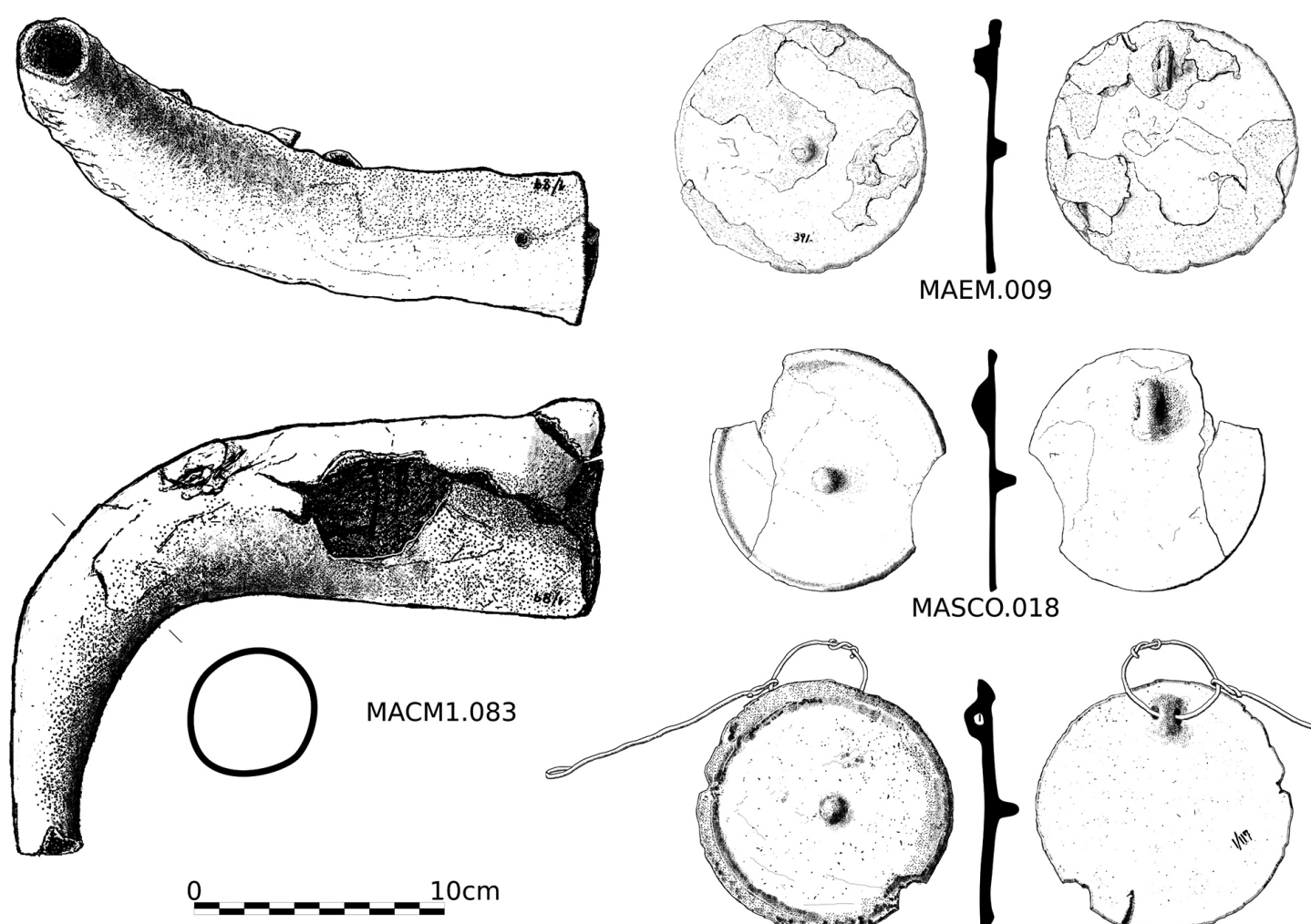

MASCO.018

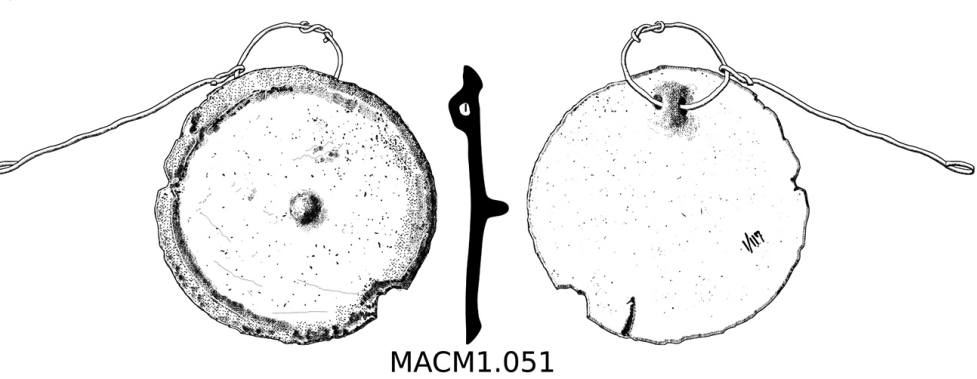

FIG. 10. Imagen de algunos de los metales analizados en este trabajo: cuerno de bronce y discos de tintinnabula.

de piezas normalmente aplanadas con decoración geométrica en el anverso y apéndices o agujeros de suspensión en el reverso (Enseñat, 1975). Se les atribuye una cronología del s. III a. C. para su aparición y podemos estar completamente seguros de su origen local, ya que no se encuentran paralelos foráneos y las evidencias de su fabricación están constatadas a través de los moldes bivalvos documentados tanto en Menorca (De Nicolás, 1988) como en Mallorca (Enseñat, 1975). En este estudio, se exponen los análisis de varias piezas procedentes de Son Espases (Figs. 6-8 y 11). Este yacimiento en concreto tiene la particularidad de ser un campamento romano datado en los primeros momentos de la ocupación $-c$. s. I a. C. - y situado a pocos metros de un asentamiento autóctono. Los ejemplares de plaquitas de plomo fueron recuperados entre un numeroso conjunto de estos objetos típicamente postalayóticos, que fue interpretado por los directores de la excavación como un almacenamiento de material destinado a talleres (Estarellas et al., 2014), probablemente para su reciclado.

Además de estos, otros artefactos de plomo característicos del mundo indígena son los que Veny (1947: 46) denominó 'cinturones' a partir de los hallazgos de Cometa des Morts I. Se refería así a pequeñas plaquitas troqueladas de unos pocos centímetros y de forma rectangular, que según el citado autor irían engarzadas unas a otras formando un supuesto cinturón. Estos objetos también se documentan en Son Real -Santa Margalida- (Hernández-Gasch, 1998: 86), Ses Copis, Son Julià, Son Cresta, Son Bosc (Enseñat, 1981) y Son Maiol'5. Se ha interpretado ${ }^{6}$ que podría ser un tipo de producción que empezaría a darse antes del s. III a. C. Hernández-Gasch (1998) confirma esta cronología para

5 Coll, J.: La evolución del ritual funerario en la Cultura Talayótica, pp. 257-258. Tesis doctoral inédita presentada en 1989 en la Univ. de les Illes Balears.

6 Coll, op. cit. n. 5, p. 456. 


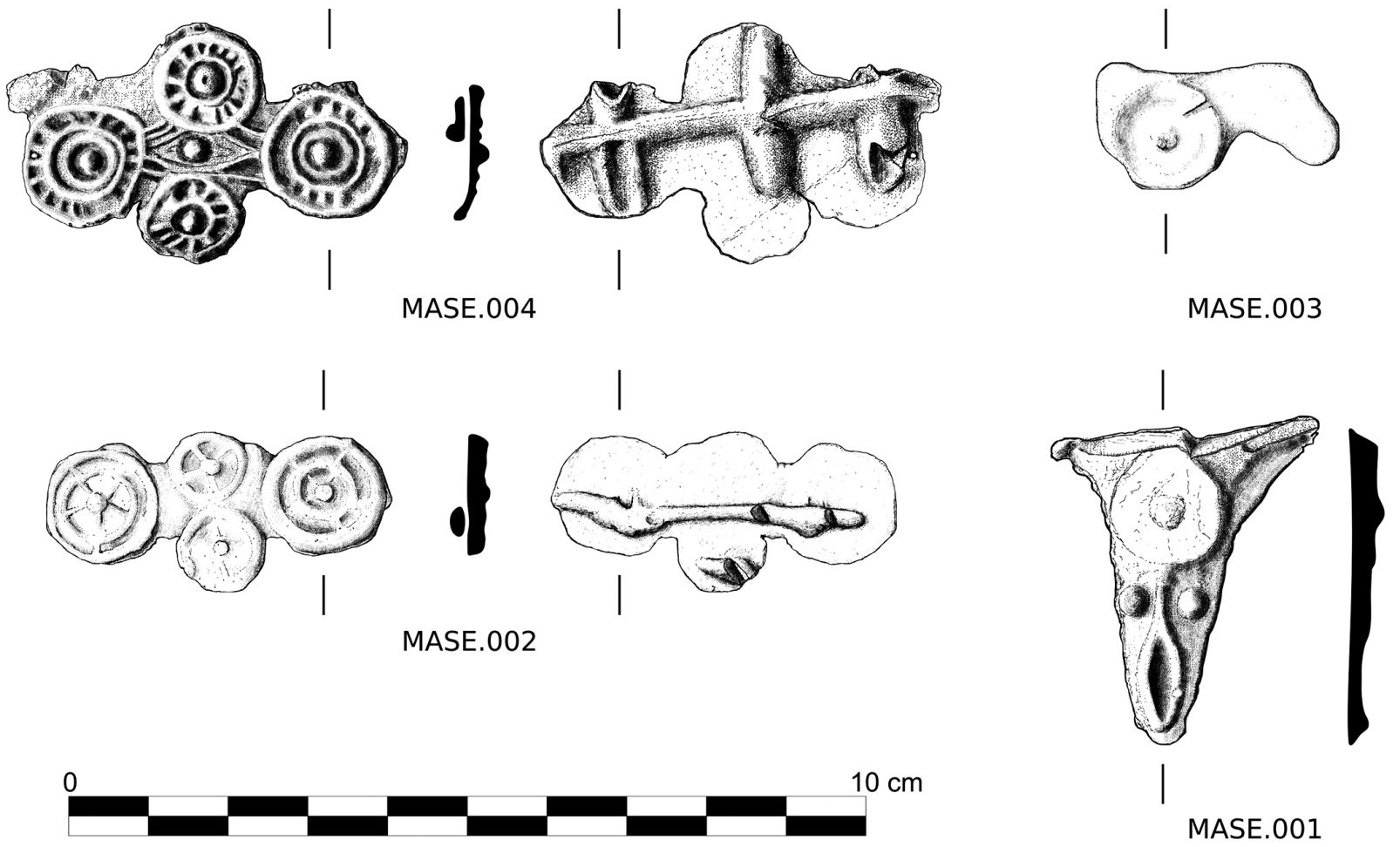

Fig. 11. Plaquitas de plomo de Son Espases analizadas en este trabajo.

los ejemplares aparecidos en Son Real en contextos de en torno al s. Iv a. C. De esta última tipología es una pieza analizada de Son Real para el presente estudio -SR14-. Además, procedente de este mismo yacimiento, también se presentan los análisis de un objeto indeterminado de plomo $^{7}$ (Fig. 9), parecido a un aplique -SR15-.

Para acabar, entre los objetos analizados que se presentan en este artículo, hay una doble hacha votiva procedente de Son Maimó, Petra, y un cuerno de bronce de Cometa des Morts I, Escorca, ambas piezas aleaciones ternarias. Este tipo de objetos, al igual que los anteriores, también pueden encontrarse en Menorca.

Hernández-Gasch, J.; Burgaya, B. y Munar M.: Intervenció Arqueologica a la necròpolis de Son Real (Cementiri dels Fenicis), Santa Margalida, Mallorca. Anys 2012-2017. Memoria plurianual inédita depositada en 2017 en el Dpto. de Cultura, Patrimoni i Esports, Consell de Mallorca.

\subsection{Discusión}

De la comparación isotópica de los objetos mencionados con los de menas de plomo actualmente disponibles en publicaciones y bases de datos, podemos extraer algunas interesantes conclusiones acerca de su posible procedencia. Para ello, aquí se hacen comparaciones con minerales de Ibiza (Ramon et al., 2011; Hermanns, 2014b), Murcia (Graeser y Friedrich, 1970; Arribas y Tosdal, 1994; Stos-Gale et al., 1995; Klein et al., 2009), Cataluña (Canals y Cardellach, 1997; Montero et al., 2009; Montero, 2017), Túnez (Jemmali et al., 2011, 2014; Jemmali et al., 2013), Linares (Tornos y Chiarada, 2004; Santos et al., 2004; Dayton y Dayton, 1986; Lillo, 1992; Hunt et al., 2011) y los minerales de las Baleares que presentamos en este trabajo (Figs. 2-3). Para tener elementos de comparación, contamos además con una serie de análisis de materiales recuperados en el yacimiento púnico-ebusitano de 


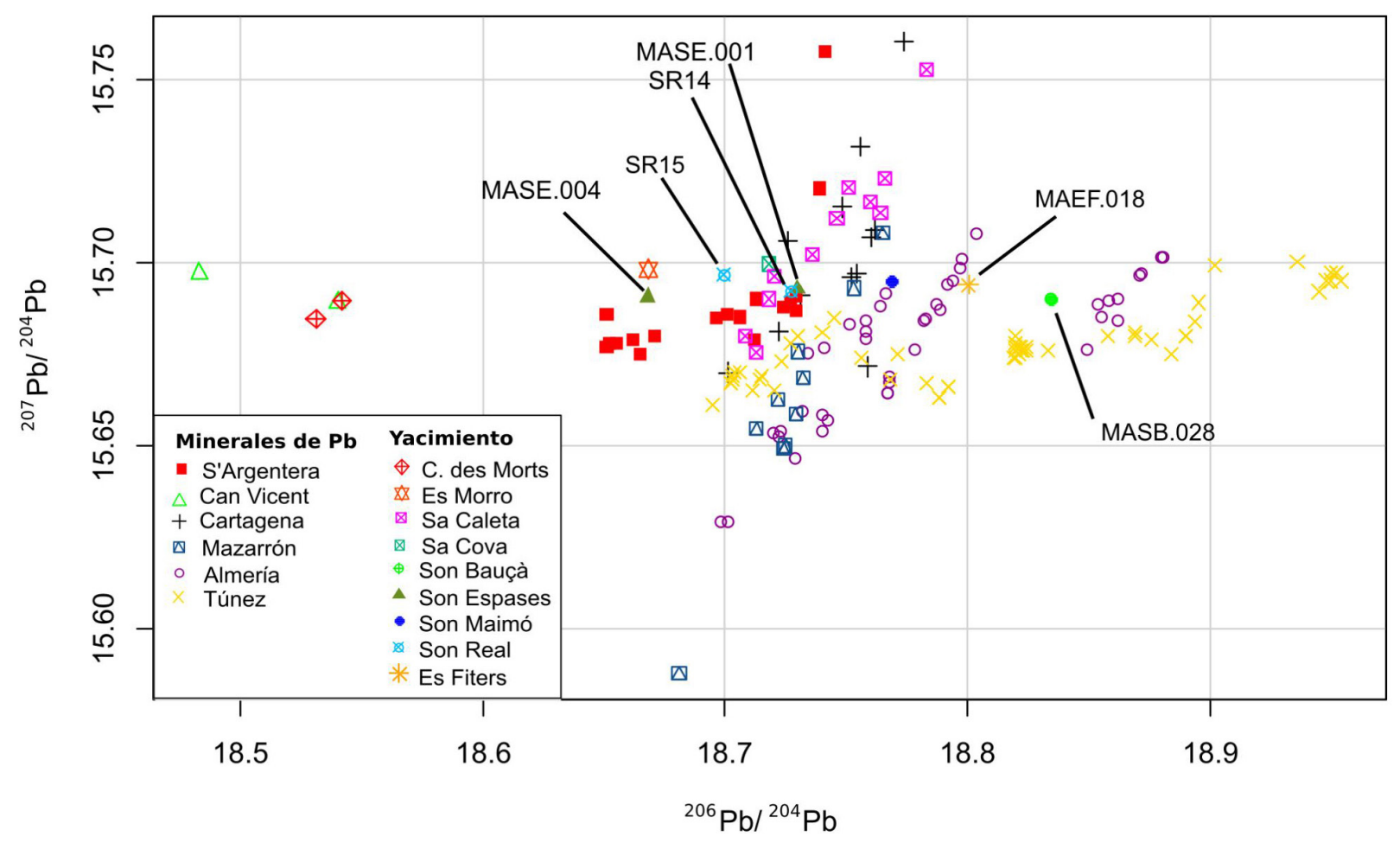

Fig. 12. Diagrama de dispersión en el que se contrastan los isótopos ${ }^{207} \mathrm{~Pb}{ }^{204} \mathrm{~Pb}$ vs ${ }^{206} \mathrm{~Pb}{ }^{204} \mathrm{~Pb}$ para las piezas con valores isotópicos próximos a las zonas de Ibiza, Cartagena, Almería y Túnez.

Sa Caleta ${ }^{8}$ (Ramon et al., 2011) con una cronología del s. vir a. C.

En primer lugar, tenemos tres piezas que coinciden plenamente con el campo isotópico definido por la mineralización ibicenca de S’Argentera. Se trata de una de las plaquitas de plomo -MASE.004procedente del yacimiento de Son Espases, una de las piezas de plomo de Son Real (SR15) y un disco de bronce ternario con un contenido en $\mathrm{Pb}$ del 4,59\% de Es Morro-MAEM.009- (Figs. 12 y 13).

Por otra parte, a pesar del solapamiento parcial de los campos isotópicos de S'Argentera y Cartagena mencionado más arriba, quedan bien definidos en el campo de Cartagena dos objetos de plomo: el SR14 de Son Real y la MASE.004 de Son Espases. En cambio, la pequeña hacha del yacimiento de Son Maimó -MACSM.001-, con un alto contenido en $\mathrm{Pb}-28,80 \%-$, parece coincidir mejor con la región de Almería, aunque, al estar en el límite del campo

8 Ruiz de Smedt, M. C., op. cit. n. 3. isotópico con Cartagena, no descartamos que también el plomo de su aleación pueda proceder de esta región o estar en la línea de mezcla entre ambas (Figs. 12 y 13). Por otra parte, la huella isotópica de la varilla de tintinnabula MAEF.018, con un 3,42\% de $\mathrm{Pb}$, parece coherente con la región de Almería en todos los contrastes. Así mismo, el plomo de la campanita de Son Bauça MASB.028 también podría proceder de esta última región, aunque al observar otros contrastes también podría encajar con mineralizaciones de Túnez, concretamente con las de Jalta (Jemmali et al., 2011, 2014) y de Jebel Hallouf (Jemmali et al., 2013). No obstante, no observamos una coincidencia determinante, haciendo que la discriminación no sea completamente segura. Este hecho también podría interpretarse como una mezcla de plomo procedente de ambas regiones (Fig. 12).

Algunos metales se concentran en el diagrama de dispersión en el espacio ocupado por los minerales de Bunyola -Arqueouib-, Binifabini Vell, Capifort y Cataluña. Algunas de las muestras que coinciden 


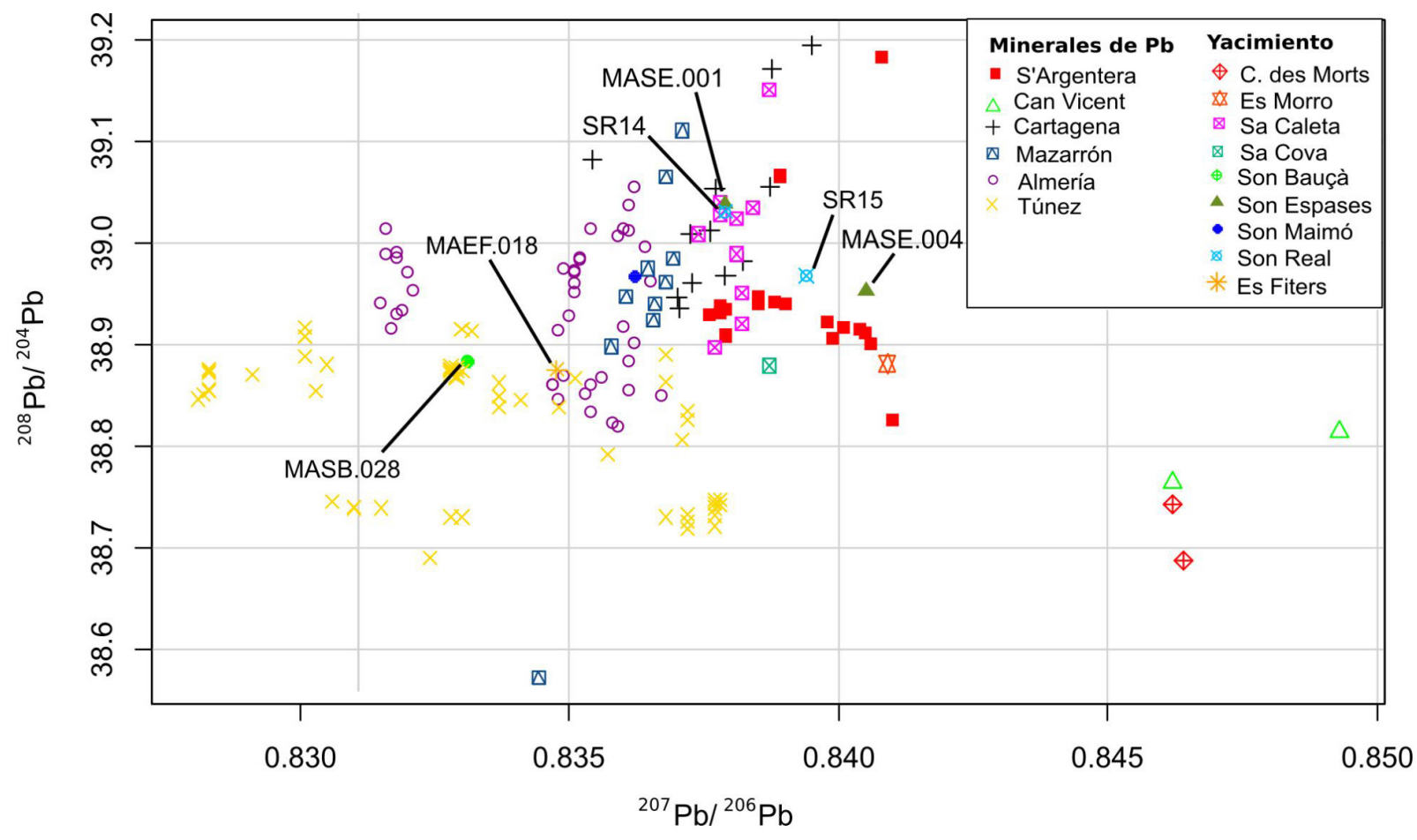

Fig. 13. Diagrama de dispersión en el que se contrastan los isótopos ${ }^{208} \mathrm{~Pb}{ }^{204} \mathrm{~Pb}$ vs ${ }^{207} \mathrm{~Pb}{ }^{206} \mathrm{~Pb}$ para las piezas con valores isotópicos próximos a las zonas de Ibiza, Cartagena, Almería y Túnez.

en esta última región de los diagramas presentan ciertos problemas de discriminación debido a los mencionados solapamientos. A pesar de esto, podemos decir que el caso de la campanita MASB.027 -Son Bauçà- parece coincidir siempre con bastante exactitud con las galenas de las Baleares en todos los contrastes. Por otra parte, el disco MAEF.017A -Es Fiters-, con un contenido en $\mathrm{Pb}$ del 22,23\%, que también parece ser coherente con estos afloramientos, observamos que se desplaza ligeramente en el contraste de los isótopos ${ }^{207} \mathrm{~Pb} /{ }^{204} \mathrm{~Pb}$ vs ${ }^{206} \mathrm{~Pb} /{ }^{204} \mathrm{~Pb}$ (Figs. 14-15), resultando un poco más coincidente con los valores isotópicos de Cataluña y haciendo más difícil su discriminación. En cualquier caso, en las gráficas generales, dichos casos se ven solapados con las galenas de Mallorca y Menorca, por lo que creemos que es completamente factible decantarnos por la posibilidad de que el plomo usado en la aleación sea local (Figs. 16-18).

En cuanto a los valores isotópicos de la campanita MASB.029 de Son Bauçà, resultan bastante coherentes con los valores de los minerales de Linares, por lo que podemos pensar en un origen de esta área geográfica para el plomo contenido en esta aleación. Sin embargo, para el caso de MASCo.018 -Sa Cova-, aunque sus valores parecen próximos a los de las galenas menorquinas, también observamos como parece situarse en los límites de los campos isotópicos de Cataluña-Mallorca-Menorca vs. Linares.

De entre todos los metales aquí analizados, hay una serie de ellos que aparentemente no encajarían en ninguno de los campos isotópicos definidos para las regiones que aquí examinamos, pero que, sin embargo, parecen coherentes con las posibles líneas de mezcla entre las zonas de Linares-Tarragona y Cartagena-Ibiza-Almería. Las piezas de Cometa des Morts I MACM1.083 у мАСм1.051 parecen próximas a las muestras de Can Vicent, en Ibiza, y en los análisis elementales se detectan trazas de $\mathrm{Ag}$ y de $\mathrm{Sb}$, especialmente en масм1.083. Sin embargo, el campo isotópico de este afloramiento no está bien definido, puesto que solo contamos con dos muestras analizadas (Hermanns, 2014b: 310). Por este motivo, aunque la procedencia más probable podría 


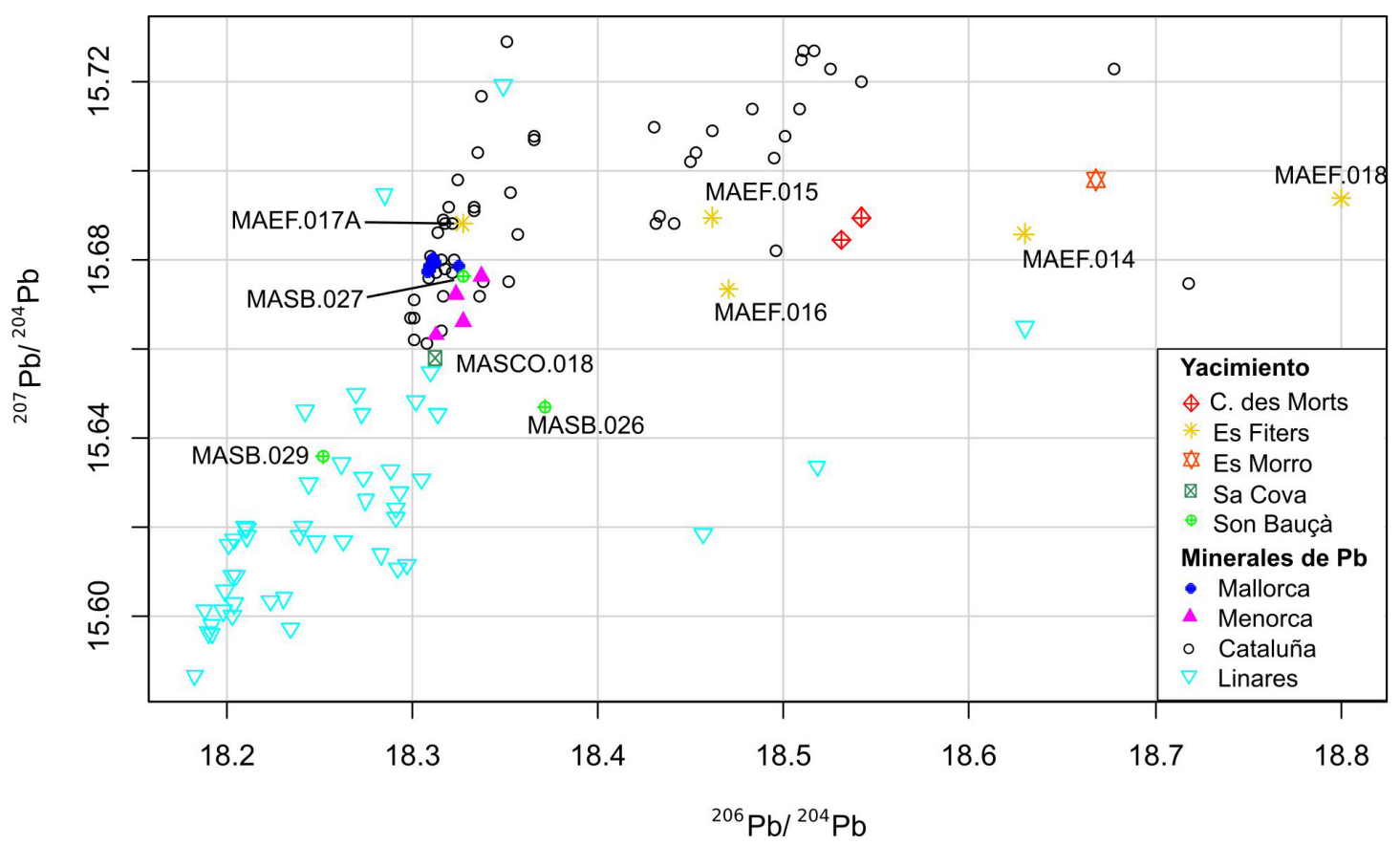

Fig. 14. Diagrama de dispersión en el que se contrastan los isótopos ${ }^{207} \mathrm{~Pb}{ }^{204} \mathrm{~Pb}$ vs ${ }^{206} \mathrm{~Pb}{ }^{204} \mathrm{~Pb}$. Pueden verse dos piezas con clara adscripción (MASB.029-Linares y MASB.027-Baleares), mientras que otros casos resultan más ambiguos.

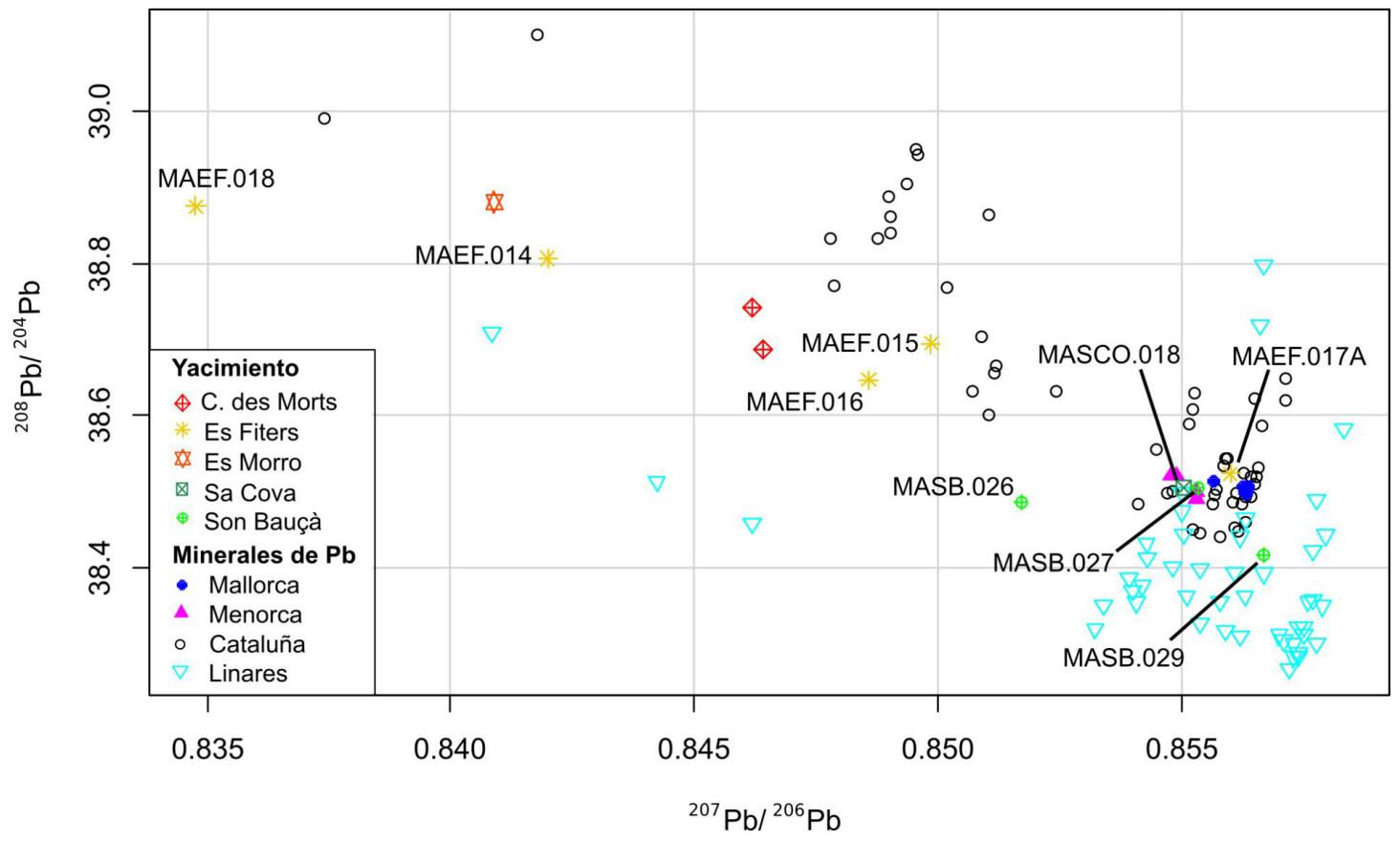

Fig. 15. Diagrama de dispersión en el que se contrastan los isótopos ${ }^{208} \mathrm{~Pb} /{ }^{204} \mathrm{~Pb}$ vs ${ }^{207} \mathrm{~Pb}{ }^{206} \mathrm{~Pb}$. Igual que en la Fig. 14, pueden verse dos piezas con clara adscripción (MASB.029-Linares y MASB.027-Baleares). En esta gráfica, MASCO.018 y MAEF.017A parecen más coherentes con los minerales de Baleares, mientras que en el contraste ${ }^{207} \mathrm{~Pb}{ }^{204} \mathrm{~Pb}$ vs ${ }^{206} \mathrm{~Pb}{ }^{004} \mathrm{~Pb}$ se mostraban más ambiguos. 
ser ibicenca, tampoco descartamos la posibilidad de que estemos ante una mezcla de metales de procedencias distintas, si bien esta mezcla sería muy semejante para estas dos piezas del mismo yacimiento. Otros objetos que parecen mostrar esta misma circunstancia son algunos de los bronces ternarios de Es Fiters, aunque en estos casos no se detectan elementos significativos en su composición: MAEF.014, MAEF.015 y MAEF.016. La campanita MASB.026 es algo más ambigua y no encontramos coincidencia plena con ninguno de los contrastes realizados, por lo que también nos inclinamos a pensar en una posible mezcla de minerales (Figs. 14-15).

\section{Circulación de metal de plomo en el contexto de los derroteros del Mediterráneo Occidental}

Una de las conclusiones importantes a las que llegan Ramon et al. (2011) es que en Sa Caleta, Ibiza, se documenta una gran cantidad de galena almacenada que no responde solo a la explotación de las minas de S'Argentera, sino que en realidad podría haber parte de este mineral originario de otros lugares destinado a ser distribuido a otras áreas del Mediterráneo. Este argumento vendría sustentado en análisis de isótopos de plomo efectuados en galenas recuperadas en Sa Caleta, que en realidad procedería de mineralizaciones cartaginesas (Ramon et al., 2011: 76). Este hecho nos ayuda a explicar algunos de los resultados de nuestros análisis. Por un lado, tenemos que algunos bronces ternarios y objetos de plomo coinciden con los campos isotópicos de las galenas de Ibiza. En cambio, una pieza de plomo de Son Espases y otra de Son Real parecen más coherentes con los minerales de Cartagena. Estos datos refuerzan la idea de Ibiza como centro redistribuidor, de manera que parte del plomo usado en bastantes metales mallorquines llegaría desde la colonia, si bien no todo tendría su origen en la propia Ebussus, sino que desde allí llegarían minerales y metales a Mallorca procedentes de otras regiones.

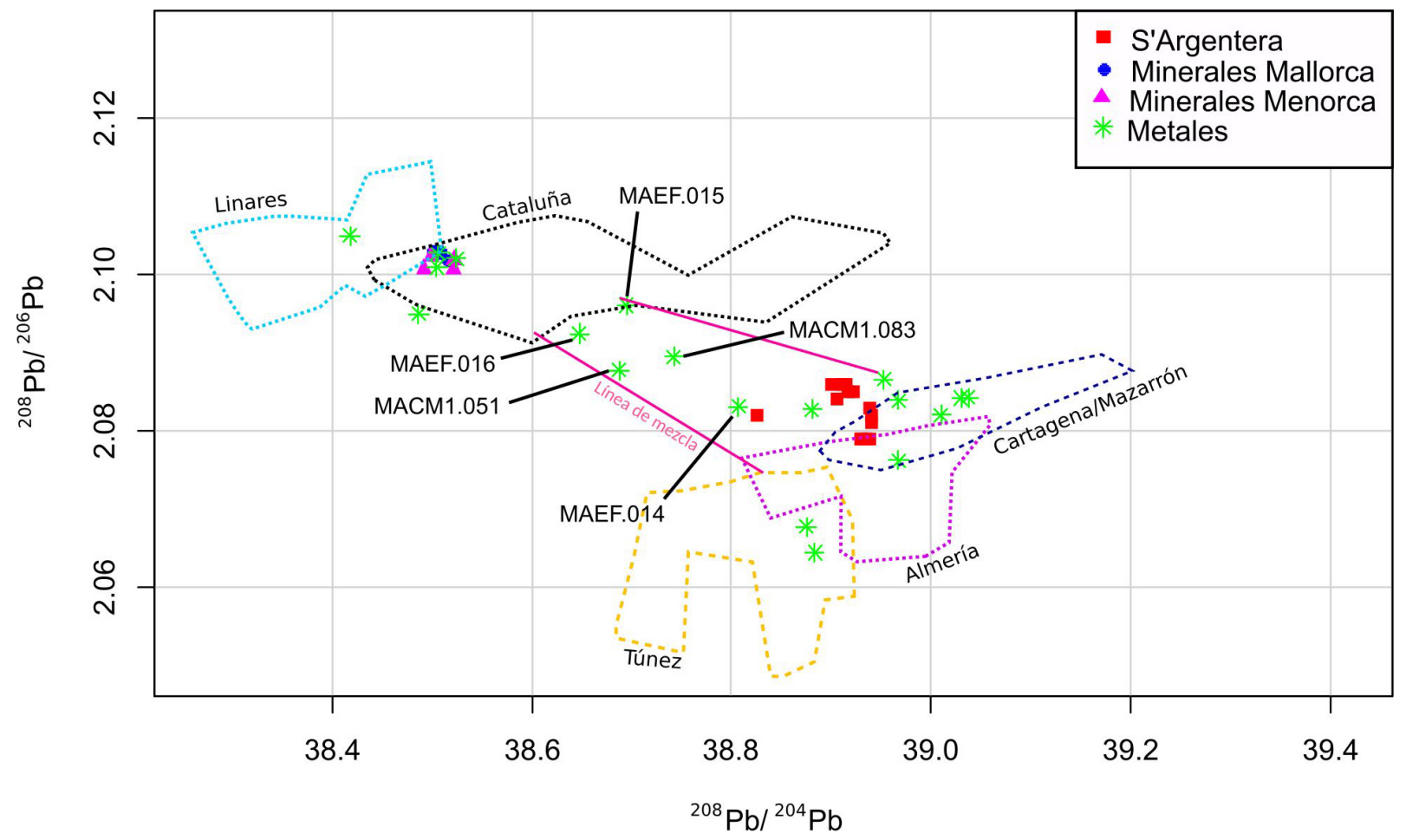

FIG. 16. Diagrama de dispersión general en el que se contrastan los isótopos ${ }^{208} \mathrm{~Pb}{ }^{006} \mathrm{~Pb} v \mathrm{~s}^{208} \mathrm{~Pb}{ }^{004} \mathrm{~Pb}$. En él pueden verse los metales que se muestran más coherentes con lineas de mezcla de diferentes regiones. 


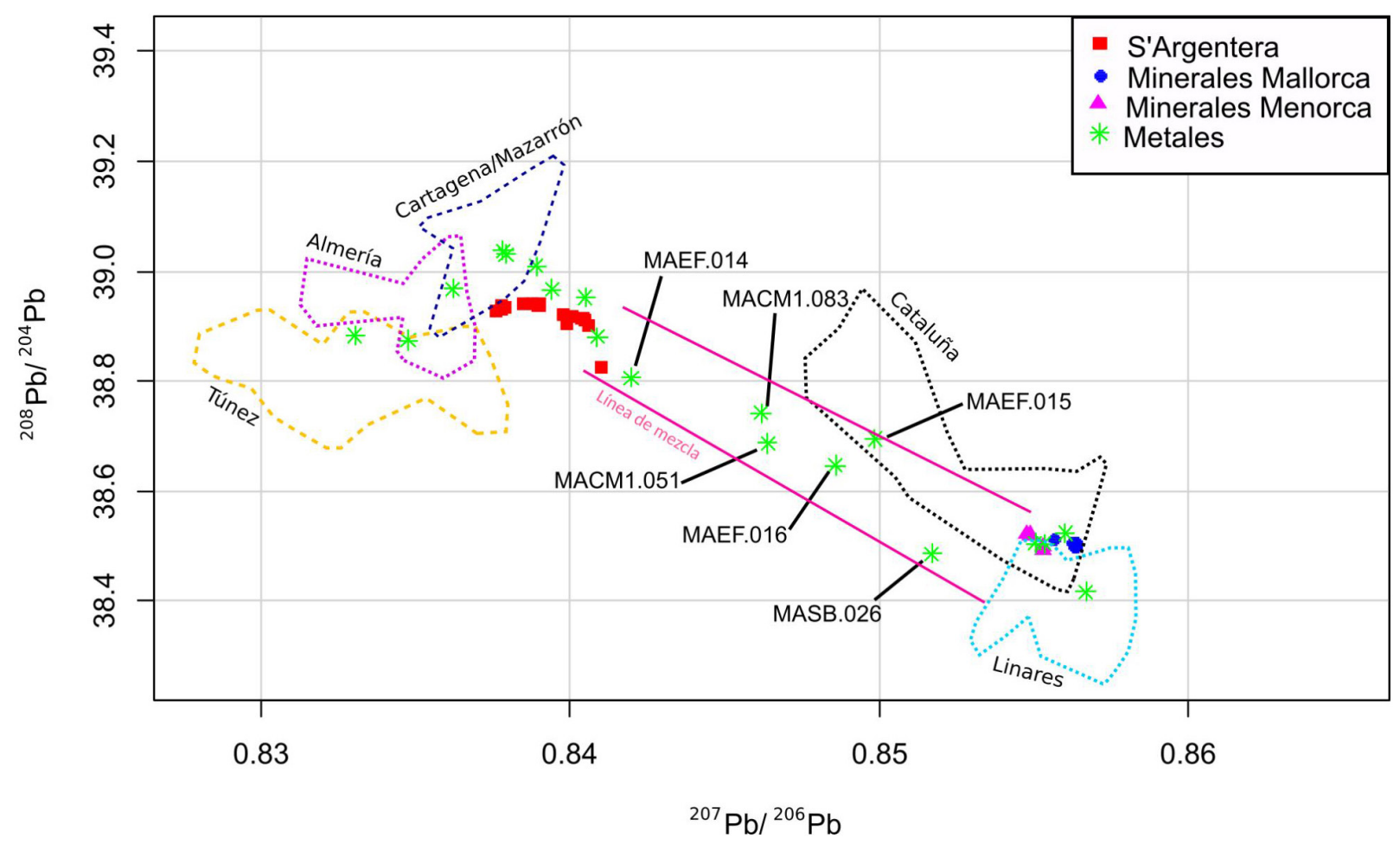

Fig. 17. Diagrama de dispersión general en el que se contrastan los isótopos ${ }^{208} \mathrm{~Pb} p^{04} \mathrm{~Pb}$ vs ${ }^{207} \mathrm{~Pb}{ }^{006} \mathrm{~Pb}$. Pueden verse los metales que se muestran más coherentes con lineas de mezcla de diferentes zonas.

Es en este contexto en el que también debemos entender la presencia de plomo procedente de la zona de Almería, Linares o tal vez del norte de África.

Conviene recordar que por ahora no hay constatación de la llegada de minerales o metales de Tarragona a Ibiza. Esto debe tenerse en cuenta si queremos contemplar la posibilidad de que los datos isotópicos de ciertas piezas presentados en este trabajo sean coherentes precisamente con las fuentes de esta área geográfica. Sin embargo, según Ramon et al. (2011), en Sa Caleta se han recuperado algunas producciones de cerámica a mano cuyo origen se encuentra posiblemente en la costa de la actual Cataluña. Entre los escasos materiales también procedentes de esta región, se encuentran dos colgantes de El Puig des Molins (Ramon et al., 2011: 66).

La otra posibilidad es que el grupo de metales coherentes con Cataluña sean en realidad plomos de Mallorca o de Menorca al estar su signatura isotópica solapada y ser prácticamente imposible de discriminar. En los diagramas, las galenas de la zona de Cataluña muestran una mayor dispersión, a diferencia de las de Mallorca y Menorca, que están más concentradas. En este sentido, los materiales metálicos analizados coincidentes con estas áreas geográficas se observan relativamente próximos, siendo en todos los casos valores muy semejantes a los minerales de Mallorca y Menorca (Figs. 16-18).

Para analizar la circulación de los metales en la protohistoria, debemos tener presentes diferentes aspectos. Por una parte, hay que distinguir entre flujo comercial y derrotero marítimo. El primero haría referencia a la procedencia de los materiales, mientras que el segundo indica la ruta que dichos materiales han recorrido para llegar a su destino (Guerrero, 2004a) $)^{9}$. Por otra parte, la navegación en la Antigüedad está condicionada en gran parte por el régimen

9 Calvo, M. y García, J.: "Mirando al mar. Revisitando las evidencias de contactos entre las comunidades indígenas y fenicio-púnicas en Mallorca (ss. VII-II ANE)". En Colloque international. Insularité, îleité, insularisation en Méditerranée phénicienne et punique 2017, en prensa. 


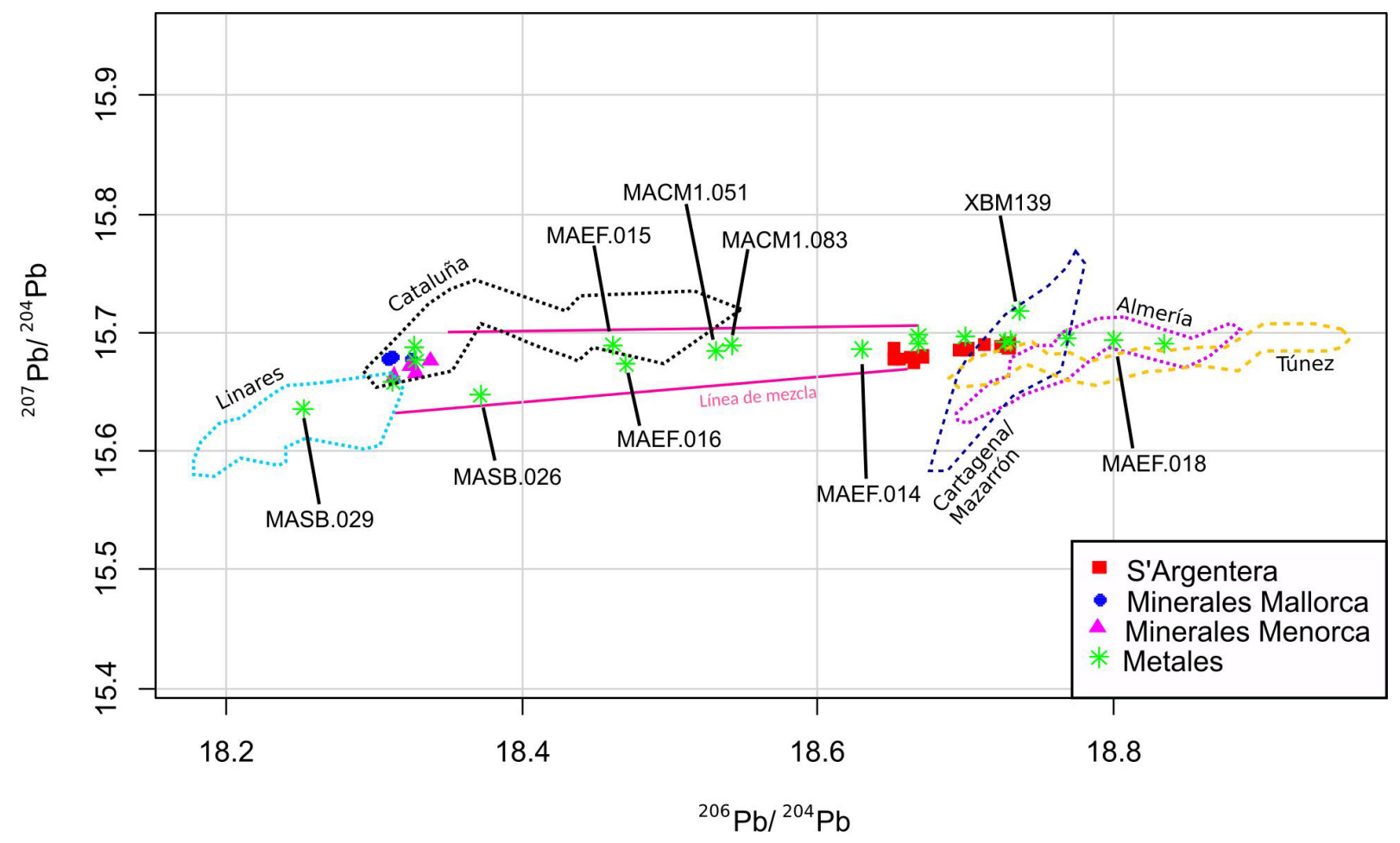

Fig. 18. Diagrama de dispersión general en el que se contrastan los isótopos ${ }^{207} \mathrm{~Pb}{ }^{204} \mathrm{~Pb}$ vs ${ }^{206} \mathrm{~Pb}{ }^{204} \mathrm{~Pb}$. Pueden verse los metales que se muestran más coherentes con líneas de mezcla de diferentes zonas.

de vientos y de corrientes, además de las condiciones meteorológicas y marítimas. Esta circunstancia puede seguirse hasta más o menos el s. II a. C., por lo que, a pesar de los progresos en la tecnología naval, apenas cambiaron los derroteros que comunicaban los diferentes puntos del mar Mediterráneo (Guerrero, 2004b: 109). Por este motivo, además de la presencia o ausencia de materiales en un sitio concreto, conviene analizar cuál es la singladura más favorable para los navegantes de estas cronologías.

Guerrero (2004b: 112) propone diferentes circuitos de navegación, que variarían en función de los vientos, las corrientes, así como de condiciones climatológicas concretas. Uno de estos tramos propuestos seguiría una navegación costera desde Carthago que iría ganando latitud hasta alcanzar Ibiza. Otra opción sería la de continuar por la costa de Argelia y desde allí llegar a una altura próxima al cabo de Gata.

Los plomos de Linares podrían haber llegado a las costas levantinas a través de alguna ruta por tierra, aunque no podemos descartar una vía fluvial bajando el Guadalquivir y llegando a la costa atlántica (Montero et al., 2012). Desde allí, cruzando el Estrecho, llegaría a las costas de Almería y doblaría el cabo de Gata. En cualquier caso, el plomo procedente de Cartagena, Linares, Almería o Túnez podría haber llegado a las Baleares a través de un mismo tramo final del derrotero, alejándose de la costa a una altura próxima al cabo de Palos y dirigiéndose hacia Ebussus. Si consideramos la posibilidad de que el plomo con valores coherentes con Tarragona proceda realmente de esta zona, debemos tener en cuenta un derrotero diferente. Se llegaría a la altura del cabo de la Nao mediante navegación de cabotaje, desde donde puede observarse la silueta de Ibiza sin perder de vista la costa peninsular (Guerrero, 2004b: 112). Esta ruta es, además, coherente con las evidencias de plomo procedente de Cataluña en el Suroeste peninsular, donde se utilizaría para copelación (Murillo-Barroso et al., 2016; Rafel et al., 2019). Por otra parte, la navegación entre islas es 
siempre de cabotaje. Se trataría de tramos cortos. La conexión entre Ibiza, Mallorca y Menorca se efectuaría por la vertiente más meridional, hecho que parecen evidenciar los datos arqueológicos, puesto que en estas áreas costeras de Mallorca es donde se encuentra mayor variedad y cantidad de materiales foráneos con una amplia continuidad en el tiempo ${ }^{10}$.

\section{Conclusiones: más allá de las procedencias}

A partir de los datos presentados en este trabajo, parece que tendríamos algunos casos de plomo procedente de Ibiza, y otros que se corresponderían bien con Linares, Cartagena, Almería o tal vez Túnez. Dichos resultados nos están definiendo un paisaje claramente de ámbito fenicio, donde la mayoría de las áreas señaladas, ricas en recursos de plomo, se encuentran controladas por estos o bajo su influencia. Además, sabemos que desde Ebussus se estaba redistribuyendo plomo de otras regiones, como atestiguan los análisis isotópicos de Sa Caleta (Ramon et al., 2011). Aunque los datos de dicho yacimiento nos están informando de la situación en cronologías anteriores a las que aquí barajamos, pensamos que estas redes permanecen vigentes, al menos hasta la Segunda Guerra Púnica. Generalmente, los investigadores coinciden en identificar como púnicos a los agentes que habrían hecho llegar a Mallorca la mayoría de los materiales foráneos durante la segunda Edad de Hierro, atribuyendo a Ebussus un destacado protagonismo. Además, debemos tener presente que un navío podía llevar cargamento de múltiples procedencias, y que estas no tenían por qué coincidir con su identidad (Guerrero, 2009-2010: 140). En este caso, parece claro que esta atribución estaría bien sustentada, ya que el tráfico de los recursos identificados en los valores isotópicos estaría en estas cronologías controlado, directa o indirectamente, por los agentes fenicio-púnicos.

Como hemos mencionado más arriba, el posible origen del metal en el No peninsular parece más difícil de concretar, ya que existe una alta similitud de las muestras de los objetos a las galenas de las

10 Calvo, M. y García, J., op. cit. n. 9. islas, además de la escasez de materiales arqueológicos documentados procedentes de esta región de la Península. Ciertamente, el material anfórico ibérico recuperado en Mallorca es muy escaso en comparación con otros productos de importación y, particularmente, la muestra de ánforas procedentes de la actual Cataluña tiene muy poca representación (Tsantini et al., 2018). Este hecho podría reforzar la idea de la utilización de minerales locales para estos objetos. Sin embargo, cabe la posibilidad de que este plomo con origen en el No hubiera llegado a las Baleares desde el so, donde este elemento era necesario para la copelación. Esta podría ser una explicación a la posible presencia de plomo procedente de esta región y, a la vez, a la ausencia de otros materiales arqueológicos.

Igualmente, debemos decir que, durante los primeros contactos fenicios con las Baleares, se detecta una demanda selectiva de productos por parte de las comunidades indígenas. Así, los objetos más comunes que parecen llegar son principalmente metales y materiales exóticos, y el material anfórico hace presencia en momentos más tardíos, hacia el s. vi a. C. (Guerrero et al., 2002). Estos materiales se generalizan posteriormente de forma heterogénea en el territorio isleño, siendo los asentamientos interiores los que menos restos de cerámica a torno proporcionan (Hernández-Gasch y Quintana, 2013). De esta manera, la ausencia de materiales de una determinada procedencia no invalida la posibilidad de que lleguen otro tipo de bienes. Así y todo, sin obviar lo expuesto en las líneas anteriores, nos decantamos, como explicación más plausible para estos casos principalmente -MASCO.018 у MASB.027-, por el uso de metal de procedencia balear por dos razones: es un recurso más cercano y los valores isotópicos de estos metales concretos son más parecidos a los valores de los minerales mallorquines y menorquines. Sea como fuere, en general, parece que nos encontraríamos ante comunidades indígenas que, salvo en momentos puntuales, están haciendo uso de plomo mayoritariamente foráneo -o mezclado a través de reciclaje- para realizar objetos propios y exclusivos de sus tradiciones locales. Así, los datos nos dibujan un panorama en el que las poblaciones indígenas 
del postalayótico mallorquín están completamente integradas en los circuitos de intercambio del mundo fenicio-púnico.

La serie analítica, que aparentemente no coincide con ningún campo isotópico definido, se muestra, sin embargo, coherente con diferentes posibles líneas de mezcla entre las zonas mineras tratadas en este trabajo (Figs. 16-18). Si tenemos en cuenta el hallazgo, tanto en contextos fenicio-púnicos de habitación como en pecios, de tortas de litargirio y copelas destinadas a procesos metalúrgicos ${ }^{11}$, no es de extrañar que subproductos de copelación de distintas procedencias acaben transformados conjuntamente a su forma metálica en las zonas de redistribución. Hay que destacar que la mayoría de las analíticas elementales de las piezas de plomo mallorquinas muestran la inexistencia de impurezas de $\mathrm{Cu}, \mathrm{Ag}$ y $\mathrm{Sn}$-siempre teniendo en cuenta las limitaciones del equipo de medición-, con lo cual, podríamos deducir que se trata de plomos no argentíferos bastante puros o bien del resultado de plomos obtenidos durante la copelación. Sin embargo, no hay que descartar el reciclado de objetos metálicos de distintas procedencias, como parece indicar la analítica de la plaquita de plomo MASE.002 que nos muestra un $1,25 \%$ de $\mathrm{Sn}$ : probablemente resultado de haber acabado alguna pequeña pieza de Sn entre otros metales de plomo para su fusión, aunque no podemos saber si el contexto de dicha actividad pirometalúrgica fue fenicio-púnico o indígena.

Por otra parte, es destacable el hecho de que las procedencias varias del plomo consumido o utilizado por las poblaciones indígenas no parecen tener correlación con los yacimientos estudiados, de manera que no vemos una posible inclinación por el metal de una determinada procedencia por parte de unas u otras comunidades. Teniendo en cuenta que los datos isotópicos son todavía escasos, y que con el material cerámico sí que han podido hacerse inferencias consistentes en este sentido, creemos que debemos ser prudentes y esperar a que se amplíe el

11 Renzi, M.: La Fonteta (Guardamar del Segura, Alicante) y la metalurgia fenicia de época arcaica en la Peninsula Ibérica, pp. 83-83. Tesis doctoral inédita presentada en 2012 en la Univ. Complutense de Madrid. número de muestras analizadas. De todos modos, si admitimos la función de Ebussus como centro redistribuidor del plomo que llega a Mallorca y Menorca, probablemente la percepción de las poblaciones indígenas sería la de un plomo de origen único.

La gran cantidad de plomo puesta en circulación como subproducto de la copelación pudo haber provocado la aparición y evolución de gustos locales a partir del aprovechamiento de este 'abundante' metal. Tal como se ha interpretado para los contextos del Priorat (Rafel et al., 2019), una consecuencia de la demanda de plomo generada por parte de los fenicios es que se pudo haber activado una explotación indígena de los recursos de galena locales para destinarlos a este 'mercado'. Si ocurrió de este modo en el archipiélago, esto pudo haber originado, con el tiempo, una demanda interna de dicho metal, y quizás en este contexto podamos encontrar la respuesta a la aparición de nuevos y abundantes objetos como las plaquitas de plomo.

Una parte del plomo utilizado podría ser tanto mallorquín como menorquín. Las ratios isotópicas de ambas islas son muy similares, y algunos de los metales analizados por nosotros parecen ser coherentes con estos minerales. De ser así, si estamos en lo cierto en cuanto a la procedencia local del plomo contenido en estos objetos, vale la pena destacar que los recursos minerales estarían controlados por las comunidades indígenas, ya que no existe ocupación territorial por parte de los agentes púnicos, al menos en el interior de las islas: la existencia de algunos asentamientos en enclaves estratégicos costeros como $\mathrm{Na}$ Guardis no supone un control territorial de la isla, ni la subordinación de las comunidades locales. Esta misma interpretación se propone para las explotaciones de plomo del Noroeste peninsular (Rafel et al., 2019).

Esto significa que la distribución interna de estos minerales o metales también estaría probablemente en manos de las poblaciones locales. El planteamiento de que hubiese en funcionamiento redes de intercambio y comunicación simultáneas es completamente compatible con las propuestas de otros autores basadas en los estudios de cerámicas de importación (Hernández-Gasch y Quintana, 
2013) ${ }^{12}$, de los que se deduce que las situaciones de contacto e intercambio fueron heterogéneas, $y$ en los que se reconoce un papel activo de las comunidades indígenas en cuanto a la redistribución de estos materiales en el interior de la isla. Todo esto nos dibuja un panorama complejo, en el que existirían diferentes niveles de circulación de materiales.

\section{Bibliografía}

Arribas, A. y Tosdal, R. M. (1994): "Isotopic composition of $\mathrm{Pb}$ in ore deposits of the Betic Cordillera, Spain; origin and relationship to other European deposits”, Economic Geology, 89 (5), pp. 1074-1093.

Calvo, M.; Quintana, C. y Guerrero, V. (2009): "Territori i intercanvi: la influencia púnica en la comunitat talaiòtica del Puig de sa Morisca (Calvià)". En SALAS, M. (coord.): I Trobada d'arqueòlegs de les Illes Balears. Manacor: Consell de Mallorca, pp. 63-72.

Canals, A. y Cardellach, E. (1997): "Ore lead and sulphur isotope pattern from the low temperatures veins of the Catalonian Coastal Ranges (NE Spain)", Mineralium Deposita, 32, pp. 243-249.

Dayton, J. E. y Dayton, A. (1986): "Uses and limitations of lead isotopes in archaeology". En Olin, J. S. y Blackmann, M. J. (eds.): Proceedings of the 24th International Archaeometry Symposium. Washington DC: Smithsonian Institution Press, pp. 13-41.

De Nicolás, J. C. (1988): "El jaciment funerari de sa Cova dels Ossos (sa Torre Nova, es Migjorn) i la metal-lúrgia del plom als darrers segles de la cultura talaiòtica de Menorca”, Meloussa, 1, pp. 9-52.

Encinas, J. A. (2014): Corpus Cavernario Mayoricense. Pollença: El Gall Editor.

Enseñat, C. (1975): "Las plaquetas de plomo mallorquinas: sistematización tipológica”, Mayurqa, 14, pp. 63-117.

ENSENAAT, C. (1981): Las cuevas sepulcrales mallorquinas de la Edad del Hierro. Excavaciones Arqueológicas en Espańa, 118. Madrid: Ministerio de Cultura.

Estarellas, M.; Merino, J. y Torres F. (2014): Les excavacions a Son Espases. Palma: Consell Insular de Mallorca.

GaLmÉs, A. (2015): "Visibilidad y percepción en la construcción de un paisaje prehistórico. El caso de Calviá (Mallorca, Islas Baleares)", Complutum, 26 (1), pp. 173-188.

12 Calvo, M. y García, J., op. cit. n. 9.
Graeser, S. y Friedrich, G. (1970): "Zur Frage der Altersstellung und Genese der Blei-Zink-Vorkommen der Sierra de Cartagena in Spanien", Mineralium Deposita, 5 (4), pp. 365-374.

Guerrero, V. M. (2004a): "Colonos e indígenas en las Baleares prerromanas”. En Fernández, J. H. y CosTA, B. (eds.): Colonialismo e interacción cultural: El impacto fenicio púnico en las sociedades autóctonas de Occidente. Eivissa: Treballs del Museu Arqueològic d'Eivissa i Formentera, pp. 145-203.

Guerrero, V. M. (2004b): "Las islas Baleares en las rutas de navegación del Mediterráneo central y occidental". En Peña, V.; Mederos, A. y Wagner, C. G. (eds.): La Navegación Fenicia: Tecnología Naval y Derroteros. Madrid: Centro de Estudios Fenicios y Púnicos-uCM, pp. 85-134.

Guerrero, V. M. (2009): “¿Foceos en el comercio tardoarcaico al norte de Baleares?”, Mayurqa, 33, pp. 131-160.

Guerrero, V. M.; Calvo, M.; García Rosselló, J. y Gornés, S. (2007): Prehistoria de las Islas Baleares. Registro arqueológico y evolución social antes de la Edad del Hierro. BAR Intern. Ser., 1690. Oxford: Archaeopress.

Guerrero, V. M.; Calvo, M. y Salvà, B. (2002): "La cultura talayótica. Una sociedad de la Edad del Hierro en la periferia de la colonización fenicia”, Complutum, 13, pp. 221-258.

Hermanns, M. H. (2014a): "Avances en el estudio histórico de la mina de galena de Bunyola (isla de Mallorca)", Saguntum, 46, pp. 189-200.

Hermanns, M. H. (2014b): "La zona minera de S'Argentera, Isla de Ibiza (Islas Baleares)", Cuadernos de Prehistoria y Arqueología de la Univ. de Granada, 24, pp. 301-318.

Hermanns, M. H. (2015): “Las minas de S'Argentera: explotación de galena de época prerromana en Ibiza”. En López, J. M. (coord.): Phicaria III. Minería y metalurgia en el Mediterráneo y su periferia oceánica. Mazarrón: Univ. Popular de Mazarrón, pp. 265-278.

Hernández-Gasch, J. (1998): Son Real. Necrópolis talayótica de la edad del hierro. Estudio arqueológico y análisis social. Arqueomediterránia, 3. Barcelona: Univ. de Barcelona.

Hernández-Gasch, J. y Quintana, C. (2013): "Cuando el vino impregnó la isla de Mallorca: el comercio púnico-ebusitano y las comunidades locales durante la segunda mitad del siglo v y el siglo IV a. C.", Trabajos de Prehistoria, 70 (2), pp. 315-331.

Hunt, M. A.; Contreras, F. y Arboledas, L. (2011): "La procedencia de los recursos minerales metálicos en el poblado de la Edad de Bronce de Peñalosa 
(Baños de la Encina, Jaén). Resultados de análisis de isótopos de plomo". En Mata-Perelló, J. M.; ToRró, L.; Fuentes, N.; Neira, A. y Puche, O. (eds.): Actas Quinto Congreso Internacional sobre Mineria y Metalurgia Históricas en el Suroeste Europeo (León2008). Homenaje a C. Domergue. Lleida: SEDpgyn, pp. 195-206.

Jemmali, N.; Souissi, F.; Carranza, E. J. M. y VenNEMANN, T. W. (2013): "Sulfur and Lead Isotopes of Guern Halfaya and Bou Grine Deposits (Domes zone, Northern Tunisia): Implications for Sources of Metals and Timing of Mineralization”, Ore Geology Reviews, 54, pp. 17-28.

Jemmali, N.; Souissi, F.; Carranza, E. J. M.; VenneMANN, T. W. y Bogdanov, K. (2014): "Geochemical Constraints on the Genesis of the Pb-Zn Deposit of Jalta (Northern Tunisia): Implications for Timing of Mineralization, Sources of Metals and Relationship to the Neogene Volcanism", Chemie der Erde-Geochemistry, 74 (4), pp. 601-613.

Jemmali, N.; Souissi, F.; Villa, I. M. y Vennemann, T. W. (2011): "Ore genesis of Pb-Zn deposits in the Nappe zone of Northern Tunisia: Constraints from $\mathrm{Pb}-\mathrm{S}-\mathrm{C}-\mathrm{O}$ Isotopic Systems", Ore Geology Reviews, 40 (1), pp. 41-53.

Klein, S.; Domergue, C.; Lahaye, Y.; Brey, G. P. y Kaenel, H.-M. V. (2009): "The Lead and Copper Isotopic Composition of Copper Ores from the Sierra Morena (Spain)", Journal of Iberian Geology, 35 (1), pp. 59-68.

Lillo, J. (1992): "Vein-type base-metal ores in Linares-La Carolina (Spain): ore-lead isotopic constrains", European Journal of Mineralogy, 4, pp. 337-343.

Llull, B. y Perelló, L. (2013): "La mineria del coure a Menorca. De la prehistòria a l'actualitat", Bolletí de la Societat Arqueològica Lul.liana. Revista d'Estudis Històrics, 69, pp. 75-93.

Lull, V.; Micó, R.; Rinuete, C. y Risch, R. (eds.) (1999): Ideología y sociedad en la prehistoria de Menorca. La Cova des Càrritx y la Cova des Mussol. Barcelona: Consell Insular de Menorca.

Montero, I. (2010): "Tecnología de la metalurgia de base de cobre”. En Montero, I. (ed.): Manual de arqueometalurgia. Madrid: Colegio Oficial de Doctores y Licenciados en Filosofía y Letras y en Ciencias, Sección de Arqueología, pp. 159-188.

Montero, I. (2017): "The archaeometallurgical perspective". En Rafel, N.; Hunt, M. A.; Soriano, I. y Delgado-Raack, S. (eds.): Prehistoric copper mining in the north-east of the Iberian Peninsula: La Turquesa or Mas de les Moreres Mine (Cornudella de Montsant, Tarragona, Spain). Revista d'Arqueologia de Ponent, Extra 3. Lleida: Univ. de Lleida, pp. 63-71.

Montero, I. (2018): "La procedencia del metal: consolidación de los estudios con isótopos de plomo en la Península Ibérica", Revista d'Arqueologia de Ponent, 28, pp. 311-328.

Montero, I.; Gener, M.; Renzi, M.; Hunt, M.; RoVIRA, S. y Santos, J. F. (2009): "Provenance of lead in first Iron Age sites in southern Catalonia (Spain)". En Moreau. J. F.; Auger, R.; Сhabot, J. y HerzoG, A. (eds.): Proceedings $36^{\text {th }}$ International Symposium on Archaeometry (Quebec, 2006). Quebec: CELAT, pp. 391-398.

Montero, I.; Rafel, N.; Rovira, M. C.; Armada, X. L.; Graells i Fabregat, R.; Hunt, M. A.; Murrillo-Barroso, M.; Renzi, M. y Santos, M. (2012): "El cobre de Linares (Jaén) como elemento vinculado al comercio fenicio en el Calvari de el Molar (Tarragona)", Menga, 3, pp. 167-184.

Murrillo-Barroso, M.; Montero, I.; Rafel, N.; Hunt, M. A. y Armada, X. L. (2016): "The Macro-Regional Scale of Silver Production in Iberia during the First Millennium BC in the Context of Mediterranean Contacts", Oxford Journal of Archaeology, 35 (1), pp. 75-100.

Negueruela, I.; Pinedo, J.; Gómez, M.; Miñano, A.; Arellano, I. y Barba, J. S. (2000): "Descubrimiento de dos barcos fenicios en Mazarrón (Murcia)". En BARthelemy, M. y Aubet, M. E. (coords.): Actas IV Congreso Internacional de Estudios Fenicios y Púnicos (Cádiz, 1995). Cádiz: Univ. de Cádiz, 4, pp. 1671-1680.

Perelló, L. y Llull, B. (2014): "De la vitrina al contexto perdido. Explorando nuevas perspectivas en torno a los discos metálicos del Postalayótico", Materialidades. Perspectivas actuales en cultura material, 2, pp. 23-46.

Perelló, L. y Llull, B. (2019): "Música y sonoridad en los ritos funerarios del Postalayótico mallorquín", Archivo Español de Arqueología, 92, pp. 105-118.

Rafel, N.; Soriano, I.; Armada, X. L.; Hunt, M. A. y Montero, I. (2019): "Lead and copper mining in Priorat county (Tarragona, Spain): From cooperative exchange networks to colonial trade (2600-500 bC)". En Armada, X. L.; Murillo-Barroso, M. y Charlton, M. (eds.): Metals, Minds and Mobility. Integrating Scientific Data with Archaeological Theory. Oxford-Philadelphia: Oxbow Books, pp. 147-158.

Ramon, J.; Rafel, N.; Montero, I.; Santos, M.; Renzi, M.; Hunt, M. A. y Armada, X. L. (2011): "Comercio protohistórico: el registro del Nordeste peninsular 
y circulación de mineral de plomo en Ibiza y el Bajo Priorato (Tarragona)", Saguntum, 43, pp. 55-81.

Renzi, M. y Rovira, S. (2015): "Las metalurgias fenicias en el Mediterráneo". En López J. M. (coord.): Phicaria III. Minería y metalurgia en el Mediterráneo y su periferia oceánica. Mazarrón: Univ. Popular de Mazarrón, pp. 113-127.

Ricci, M. (1973): "Per una cronologia delle lucerne tardo-repubblicane", Rivista di Studi Liguri, 39, pp. 168234.

Rovira, S. (2000): Continuismo e innovación en la metalurgia ibérica. Saguntum, Extra 3. Valencia, pp. 209-221.

Salvà, B.; Llull, B.; Perelló, L. y Rovira, S. (2010): "Aproximación a la metalurgia prehistórica de las Baleares: estudio analítico de minerales cupríferos de Menorca". En Saiz, M. E.; López, R.; Cano, M. A. y Calvo, J. C. (eds.): Actas vili Congreso Ibérico de Arqueometría. Teruel: Seminario de Arqueología y Etnología Turolense, pp. 183-196.

Salvì, B.; Perelló, L. y Llull, B. (2018): "Metalurgia prehistórica en las Islas Baleares. Cambios y continuidades". En Melis, M. G. (ed.): La Préhistoire et la Protohistoire des îles de Mediterranée Occidentale. Matières premières. Sassari: Quaderni Lapars, pp. 69-98.
Santos, J. F.; García, S.; Gil, J. I. y Palero, F. (2004): "A Lead Isotope Database: The Los Pedroches-Alcudia Area (Spain); Implications for Archaeometallurgical Connections Across Southwestern and Southeastern Iberia", Archaeometry, 46 (4), pp. 625-634.

Stos-Gale, Z.; Gale, N. H.; Houghton, J. y SpeakMAN, R. (1995): "Lead isotope data from the isotrace laboratory, Oxford: Archaeometry data base 1, ores from the western Mediterranean", Archaeometry, 37 (2), pp. 407-415.

Tornos, F. y Chiaradia, M. (2004): "Plumbotectonic Evolution of the Ossa Morena Zone, Iberian Peninsula: Tracing the Influence of Mantle-Crust Interaction in Ore-Forming Processes", Economic Geology, 99, pp. 965-985.

Tsantini, E.; Quintana, C.; Albero, D. y Cau, M. A. (2018): "Iberian amphorae beyond the mainland: imports in southwestern Mallorca (Balearic Islands, Spain)", Archaeological and Anthropological Sciences. https://doi.org/10.1007/s12520-018-0630-x

Veny, C. (1947): "La necrópolis de la cueva 'Cometa dels Morts', cerca de Lluch, en Mallorca", Archivo Español de Arqueología, 20, pp. 46-59. 
